\title{
Gödel space from wrapped M2-branes
}

\author{
T.S. Levi ${ }^{1}$, J. Raeymaekers ${ }^{2}$, D. Van den Bleeken ${ }^{3}$, W. Van Herck ${ }^{4}$, B. Vercnocke ${ }^{4,5}$ \\ ${ }^{1}$ Department of Physics and Astronomy, University of British Columbia, \\ Vancouver, B.C. V6T 1Z1, Canada \\ ${ }^{2}$ Institute of Physics of the ASCR, v.v.i. \\ Na Slovance 2, 18221 Prague 8, Czech Republic \\ ${ }^{3}$ NHETC and Department of Physics and Astronomy, Rutgers University, \\ Piscataway, NJ 08855, USA \\ ${ }^{4}$ Institute for Theoretical Physics, K.U.Leuven \\ Celestijnenlaan 200D, B-3001 Leuven, Belgium \\ ${ }^{5}$ Jefferson Physical Laboratory, Harvard University, \\ Cambridge, MA 02138, USA
}

ABSTRACT: We show that M-theory admits a supersymmetric compactification to the Gödel universe of the form $\mathrm{Gödel}_{3} \times \mathrm{S}^{2} \times \mathrm{CY}_{3}$. We interpret this geometry as coming from the backreaction of M2-branes wrapping the $\mathrm{S}^{2}$ in an $\mathrm{AdS}_{3} \times \mathrm{S}^{2} \times \mathrm{CY}_{3}$ flux compactification. In the black hole deconstruction proposal similar states give rise to the entropy of a D4-D0 black hole. The system is effectively described by a three-dimensional theory consisting of an axion-dilaton coupled to gravity with a negative cosmological constant. Other embeddings of the three-dimensional theory imply similar supersymmetric Gödel compactifications of type IIA/IIB string theory and F-theory. 


\section{Contents}

1. Introduction 1

2. Sphere-wrapping M2-branes in the probe approximation 3

3. Effective three-dimensional description 5

4. Holomorphic BPS solutions $\quad 8$

5. Joining Gödel to AdS 14

6. Discussion and future directions 20

A. Other embeddings of the 3D system 22

B. Details of the supersymmetry analysis $\quad 23$

C. Embedding in 5D $\mathcal{N}=1$ supergravity 29

$\begin{array}{ll}\text { D. Israel junction conditions } & 30\end{array}$

\section{Introduction}

BPS states have played a major role in the successes of string theory, from the understanding of black hole microstates to nonperturbative checks of dualities. An interesting set of BPS states is that of supersymmetric D-branes in an $\mathrm{AdS}_{q} \times \mathrm{S}^{p}$ background (see e.g $[1,2,3,4,5]$ and references therein). Such states are of interest for the AdS/CFT correspondence in general. Furthermore, in the special case where the background geometry corresponds to the near horizon of an extremal black hole, string or ring, there are strong indications that such BPS states, formed by wrapping branes around the $\mathrm{S}^{p}$ part of the geometry correspond to black hole (string or ring) microstates [6, 7, 8, 9]. The study of such sphere-wrapping branes has so far been performed purely in the probe approximation $[3,6,7,10]$. There are however some indications that these branes strongly backreact on the background geometry, and that some of their properties can only be fully understood once these effects are properly taken into account.

In this paper we take a first step at studying the fully backreacted geometries corresponding to such wrapped branes. We will specialise to the M-theory flux compactification $\mathrm{AdS}_{3} \times \mathrm{S}^{2} \times \mathrm{CY}_{3}$ and construct supergravity solutions corresponding to M2-branes wrapped around the $\mathrm{S}^{2}$. Note however, that by taking the $\mathrm{CY}_{3}$ to be $\mathrm{T}^{6}$ or $\mathrm{K} 3 \times \mathrm{T}^{2}$ and applying 
U-dualities these solutions can be mapped to similar configurations in type IIA/IIB string theory or F-theory.

We start our search for these solutions by noting that all the dynamics can be captured by a reduction to three dimensions and performing a consistent truncation to the fields of interest. As we will discuss in detail, the problem can be brought back to studying threedimensional gravity with a negative cosmological constant, coupled to an axion-dilaton system:

$$
\frac{S_{3 d}}{2 \pi}=\frac{1}{l_{3}} \int d x^{3} \sqrt{-g}\left(R+\frac{2}{l^{2}}-\frac{1}{2} \frac{\partial_{\mu} \tau \partial^{\mu} \bar{\tau}}{\tau_{2}^{2}}\right) .
$$

To the authors' knowledge this three-dimensional theory has never before been studied in the literature. This is somewhat surprising as three-dimensional gravity with a negative cosmological constant is a surprisingly rich gravitational theory that is well explored and remains the subject of present investigations (see [11, 12] and references therein). Furthermore, the above theory without a cosmological constant was the subject of the classic paper [13], and is very closely related to F-theory.

Due to its embedding in eleven-dimensional supergravity the above bosonic action is naturally completed into a supersymmetric theory. We will show that this theory has 1/2-BPS solutions that are all locally Gödel space ${ }^{1}$ :

$$
\begin{aligned}
d s_{3}^{2} & =\frac{l^{2}}{4}\left(-\left(d t+\frac{3}{2} \frac{d x}{y}\right)^{2}+\frac{3}{2} \frac{d x^{2}+d y^{2}}{y^{2}}\right), \\
\tau & =x+i y .
\end{aligned}
$$

The full eleven-dimensional solution can be read off by substituting this metric in formula (3.4) below. Gödel space [14] has a long history, and this work provides a new supersymmetric embedding into string/M-theory. For an earlier example see [15]. More precisely our work shows that M-theory has a compactification of the form Gödel ${ }_{3} \times \mathrm{S}^{2} \times \mathrm{CY}_{3}$ that preserves 4 out of the 32 supersymmetries. For other embeddings of spaces with closed timelike curves in string theory, see e.g. [16, 17].

As the real part of the scalar field is an axion which is dual to the gauge field that is sourced by the sphere-wrapping M2-branes, the Gödel universe carries this charge. The field configuration of global Gödel space corresponds to an infinite amount of M2-charge localised in a point on the boundary, $y=\infty$.

Gödel space suffers from closed timelike curves (CTCs). We study some simple domainwall configurations, made out of smeared M2-branes, that allow us to glue Gödel space to $\mathrm{AdS}_{3}$. It was our hope that this would eliminate the CTCs as it does for a similar system dubbed the 'hypertube' [18]. Unfortunately, at present we have not been able to find a global solution that fully eliminates CTCs. We remain optimistic that a future treatment, either with another patching or a smooth resolution of the patching such as was found for the hypertube $[19,20]$ will resolve this issue. Nevertheless, the glued geometries we have found seem very interesting from the point of view of holography.

\footnotetext{
${ }^{1}$ Gödel's original spacetime was four-dimensional, but it is nothing but the direct product of a non-trivial three-dimensional spacetime with a space-like line. It is this three-dimensional spacetime that we will refer to as Gödel space.
} 
The paper is organized as follows. In the next section we review and discuss the supersymmetric properties of the wrapped M2-brane states that motivate our study. In Sec. 3 we then detail our flux compactification of M-theory to three dimensions and how solutions sourced by the wrapped branes correspond to effectively three-dimensional geometries with a non-trivial axion-dilaton profile. In Sec. 4, we solve the equations of motion of the three-dimensional theory and show these solutions are supersymmetric. Sec. 5 covers how the Gödel space can be supersymmetrically glued to AdS space through the introduction of an appropriate domainwall. Sec. 6 presents some discussion and suggestions for future directions. For the reader's convenience we have provided some appendices containing extra technical details. Appendix A describes in some more detail the U-dualities that link our solutions to similar ones in dual frames. The supersymmetry of the Gödel solutions in eleven- and five-dimensional supergravity is carefully shown in full detail in Appendix B and Appendix C. In Appendix D we quickly review the Israel matching conditions using a rather simple approach that clarifies the generalisation to more general field theories.

\section{Sphere-wrapping M2-branes in the probe approximation}

Before embarking on the construction of backreacted solutions of $\mathrm{S}^{2}$-wrapping M2-branes, let us review what is known about these BPS objects in the probe approximation $[3,6,10]$. These properties will be useful when comparing with the backreacted solutions in Sec. 4 .

Our starting background is M-theory compactified on $\mathrm{AdS}_{3} \times \mathrm{S}^{2} \times \mathrm{CY}_{3}$, and we will assume that the anti-de-Sitter factor is global $\mathrm{AdS}_{3}$ and not a local solution such as a BTZ black hole. Such a background arises e.g. in a certain limit of a D6-anti-D6 configuration when lifted to M-theory $[8,21]$. The $\mathrm{AdS}_{3}$ part of the metric is

$$
d s_{3}^{2}=l^{2}\left[-\cosh ^{2} \rho d \sigma^{2}+d \rho^{2}+\sinh ^{2} \rho d \psi^{2}\right]
$$

and the bosonic symmetry group of the eleven-dimensional background is $S L(2, \mathbb{R})_{L} \times$ $S U(2)_{L} \times S L(2, \mathbb{R})_{R}$.

We add to this background a probe M2-brane (or anti-M2-brane) wrapped on $\mathrm{S}^{2}$, which behaves as a massive point particle in $\mathrm{AdS}_{3}$. As can be seen from (2.1), we cannot place a static particle (with respect to the global time $\sigma$ ) at finite $\rho$, as it experiences a gravitational potential and will fall towards $\rho=0$. A spinning particle however, obeying $\psi=\sigma+$ constant, can stay at any fixed constant radius $\rho=\rho_{0}$. The $\rho_{0}$-dependent momentum conjugate to $\psi$ determines the D0-brane charge after compactification on a circle to type IIA in ten dimensions. Such spinning M2-branes are BPS states and are the objects we will study, see Fig. 1.

It will be convenient to introduce a new time coordinate with respect to which the M2-brane is at rest; if we define $\sigma=t / 2, \psi=t / 2-\varphi$, our probe brane is static with respect to $t$. The metric in these coordinates becomes

$$
d s^{2}=\frac{l^{2}}{4}\left[-(d t+2 \sinh \rho d \varphi)^{2}+4 d \rho^{2}+\sinh ^{2} 2 \rho d \varphi^{2}\right] .
$$

Note that the $\mathrm{AdS}_{3}$ metric is now written as a timelike fibration over Euclidean $\mathrm{AdS}_{2}$. The new time coordinate also has a useful group-theoretical interpretation: it is the time 

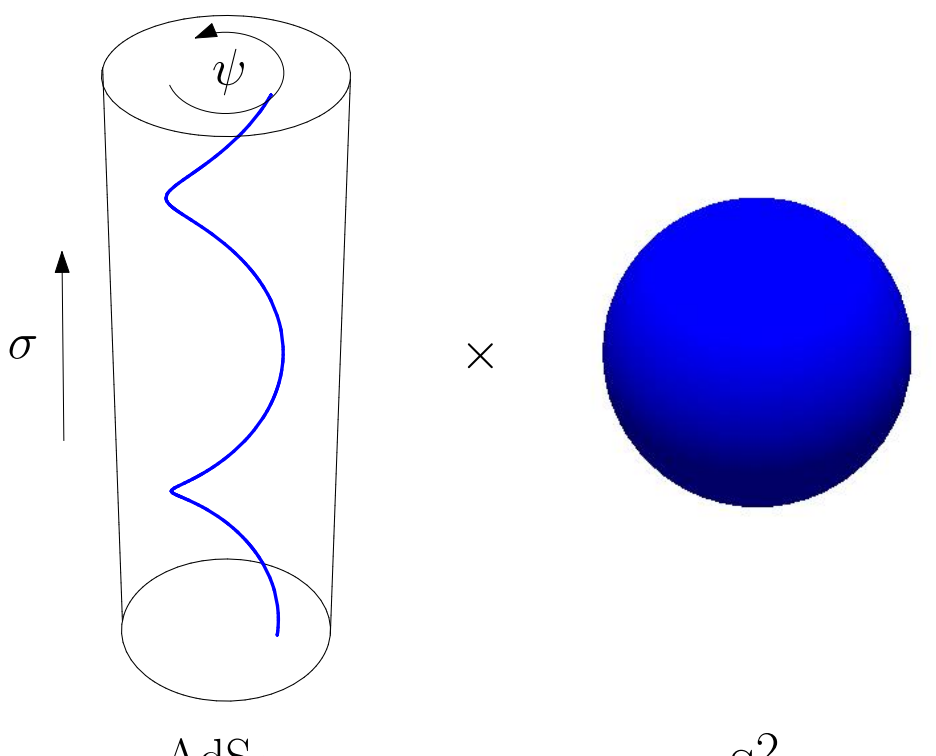

$\mathrm{AdS}_{3}$

$S^{2}$

Figure 1: The BPS M2-brane wraps the $\mathrm{S}^{2}$ and has a helical worldline, $\psi=\sigma+$ constant, in the external $\mathrm{AdS}_{3}$ space.

coordinate adapted to the $l_{0}$ generator of $S L(2, \mathbb{R})_{L}: l_{0}=\partial / \partial_{t}$. In the presence of the probe brane, the symmetry group is reduced to the generators that commute with $l_{0}$, leaving $U(1)_{L} \times S U(2)_{L} \times S L(2, \mathbb{R})_{R}$. The $l_{0}$ Noether charge is the Hamiltonian with respect to $t$, and one finds the dispersion relation

$$
L_{0}=\frac{l}{2} T_{M_{2}} \operatorname{Vol}_{S^{2}} \equiv Z,
$$

where $L_{0}$ is the eigenvalue of $l_{0}, T_{M_{2}}=2 \pi \ell_{M}^{-3}$ is the tension of the M2-brane and $l_{M}$ is the eleven-dimensional Planck length. We have used the symbol $Z$ to denote that the right-hand side is a topological charge proportional to the winding number (which we have taken to be unity) of the brane around $\mathrm{S}^{2}$; we will see below that $Z$ enters as a central extension in the worldvolume superalgebra.

The eleven-dimensional background preserves 8 fermionic symmetries which combine with $S L(2, \mathbb{R})_{L} \times S U(2)_{L}$ to form the supergroup $S U(1,1 \mid 2)_{L}$. The corresponding Killing spinors can be labelled as $g_{\frac{m}{2}}^{\alpha a}$ where the indices $m, \alpha, a$ each take the values \pm 1 . The indices $m$ and $\alpha$ are $S L(2, \mathbb{R})_{L}$ and $S U(2)_{L}$ doublet indices respectively. The explicit form of the Killing spinors can be found in (B.16, B.18). From the Killing vectors and Killing spinors one can compute the isometry supergroup of the supergravity background using the method developed in [22]. This gives the fermionic part of the of $S U(1,1 \mid 2)_{L}$ superalgebra:

$$
\begin{aligned}
\left\{g_{\frac{m}{2}}^{\alpha a}, g_{\frac{m}{2}}^{\beta b}\right\} & =\epsilon^{\alpha \beta} \epsilon^{a b} l_{m}, \\
\left\{g_{\frac{m}{2}}^{\alpha a}, g_{-\frac{m}{2}}^{\beta b}\right\} & =\epsilon^{\alpha \beta} \epsilon^{a b} l_{0}+m \epsilon^{a b} T^{\alpha \beta},
\end{aligned}
$$

where $T^{\alpha \beta}$ is an $S U(2)_{L}$ tensor given in (B.21). 
The (anti-)M2-brane probes preserve 4 out of 8 Killing spinors, as one can verify using standard methods $[23,24]$. In terms of our basis $g_{\frac{m}{2}}^{\alpha a}$, the preserved Killing spinors are

$$
\begin{array}{ll}
g_{\frac{1}{2}}^{\alpha+}, g_{-\frac{1}{2}}^{\alpha-} & \text { M2-brane, } \\
g_{\frac{1}{2}}^{\alpha-}, g_{-\frac{1}{2}}^{\alpha+} & \text { anti-M2-brane. }
\end{array}
$$

The dispersion relation (2.3) can be viewed as a BPS condition, with $Z$ playing the role of a central charge. The extended superalgebra can be computed using the methods of [25]. The result is

$$
\begin{aligned}
\left\{g_{\frac{m}{2}}^{\alpha a}, g_{\frac{m}{2}}^{\beta b}\right\} & =\epsilon^{\alpha \beta} \epsilon^{a b} l_{m}, \\
\left\{g_{\frac{m}{2}}^{\alpha a}, g_{-\frac{m}{2}}^{\beta b}\right\} & =\epsilon^{\alpha \beta} \epsilon^{a b} l_{0}+m \epsilon^{a b} T^{\alpha \beta} \mp m Z \epsilon^{\alpha \beta} \sigma_{1}^{a b},
\end{aligned}
$$

where the (plus) minus sign corresponds to a (anti-) brane. This is a centrally extended version of $S U(1,1 \mid 2)_{L}$. In the quantum theory, chiral primaries of the extended algebra saturate a BPS bound

$$
L_{0} \geq j+Z
$$

From (2.3) we see that our probes saturate this BPS bound for $j=0$ and that the preserved supersymmetries (2.5) are a consequence of the extended superalgebra.

The fact that the wrapped M2-brane is a chiral primary state in a centrally extended version of $S U(1,1 \mid 2)_{L}$ leads to a useful observation. The asymptotic symmetry algebra of the $\mathrm{AdS}_{3} \times \mathrm{S}^{2}$ background, which coincides with the superconformal algebra of the dual CFT (see $[26,27]$ for a detailed description of this algebra), does not contain the central charge term in (2.6). Therefore we can expect that the backreacted geometry of the wrapped M2-probes is no longer asymptotically $\mathrm{AdS}_{3} \times \mathrm{S}^{2}$. Another reason for expecting a large backreaction destroying the asymptotics of the background is the fact that we are dealing with charged codimension-two objects, which produce long-range fields. Indeed, we will find that, after backreaction, the $\mathrm{AdS}_{3}$ factor is to be replaced with Gödel space, which is not an asymptotically $\mathrm{AdS}_{3}$ geometry. We will construct such backreacted solutions and show that they preserve the same bosonic $U(1)_{L} \times S U(2)_{L} \times S L(2, \mathbb{R})_{R}$ symmetries and the same supersymmetries (2.5) as the probe.

\section{Effective three-dimensional description}

The arguments of the last section lead us to search for supersymmetric solutions where the fields that are sourced by the sphere-wrapped M2-branes under consideration are turned on. We will show that there is a consistent reduction on the $\mathrm{S}^{2} \times \mathrm{CY}_{3}$ that includes these fields and results in the three-dimensional action

$$
\frac{S_{3 d}}{2 \pi}=\frac{1}{l_{3}} \int d x^{3} \sqrt{-g}\left(R+\frac{2}{l^{2}}-(\mu-1) \frac{\partial_{\mu} \tau \partial^{\mu} \bar{\tau}}{\tau_{2}^{2}}\right)
$$

which will be the starting point for the analysis in the rest of the paper. 
This system describes three-dimensional gravity with a negative cosmological constant $\Lambda=-1 / l^{2}$, coupled to a complex scalar $\tau=\tau_{1}+i \tau_{2}$, which has a typical axion-dilaton type kinetic term. In the $l \rightarrow \infty$ limit this action is that of [13]. The eleven-dimensional origin of the scalar $\tau_{1}$ is as the Hodge dual of the electric field sourced by the M2-branes, while $\tau_{2}$ is the dynamic Calabi-Yau volume. We have introduced a coupling constant $\mu$ in front of the axion-dilaton action. Positive energy requires $\mu>1$, and we will see that the M-theory reduction fixes $\mu=3 / 2$, which is the value we will be interested in. Nevertheless, we will be able to construct solutions for more general $\mu$, although it is not clear whether these allow for a supersymmetric embedding.

We show in more detail how the above three-dimensional system can be derived from M-theory. We begin with the bosonic part of the eleven-dimensional supergravity (Mtheory) action. In our conventions it is given by

$$
\frac{S_{M}}{2 \pi}=\frac{1}{l_{M}^{9}} \int d^{11} x \sqrt{-g} R-\frac{1}{2 l_{M}^{3}} \int F_{4} \wedge \star F_{4}+\frac{1}{6} \int A_{3} \wedge F_{4} \wedge F_{4},
$$

where $l_{M}$ is the eleven-dimensional Planck length and $F_{4}=d A_{3}$. We will also use the M2-brane action

$$
\frac{S_{M 2}}{2 \pi}=-\frac{1}{l_{M}^{3}} \int_{\Sigma} d^{3} \xi \sqrt{-g}+\int_{\Sigma} A_{3} .
$$

We seek solutions that are sourced by M2-branes that wrap the two-sphere in the flux compactification of M-theory on $\mathrm{S}^{2} \times \mathrm{CY}_{3}$. We make a consistent reduction for such solutions using the ansatz

$$
\begin{aligned}
d s_{11}^{2} & =\tau_{2}^{-2 / 3}\left(d s_{3}^{2}+\frac{l^{2}}{4} d s_{S^{2}}^{2}\right)+l_{M}^{2} \tau_{2}^{1 / 3} d s_{C Y_{3}}^{2}, \\
F_{4} & =-\frac{\star_{3} d \tau_{1}}{l_{3} \tau_{2}^{2}} \wedge \omega_{2}+\frac{2 \pi l}{l_{M}} \omega_{2} \wedge J_{C Y_{3}} .
\end{aligned}
$$

The Hodge dual $\star_{3}$ is taken with respect to $d s_{3}^{2}$. We have furthermore parameterized the dynamic Calabi-Yau volume with a scalar $\tau_{2}$, so that in the notation above both the $\mathrm{S}^{2}$ and the $\mathrm{CY}_{3}$ part of the metric have fixed unit volume. The volume form on the unit $\mathrm{S}^{2}$ is $\omega_{2}$, and $J_{C Y_{3}}$ is the Kähler form on the unit volume Calabi-Yau.

In this ansatz we allow two contributions to the M-theory gauge field $F_{4}$ : the part along $\omega_{2} \wedge J_{C Y_{3}}$ describes the flux needed to support the background, while the first term in (3.5) is the one sourced by sphere-wrapped M2-branes. We describe this component in terms of a real scalar field $\tau_{1}$, as this is more natural from the three-dimensional point of view. It is related to a $U(1)$-potential $A$ by three-dimensional Hodge duality:

$$
d A=-\frac{\star_{3} d \tau_{1}}{l_{3} \tau_{2}^{2}}
$$

The three-dimensional Planck length $l_{3}$ and the AdS-radius $l$ are related to the elevendimensional Planck length $l_{M}$ as

$$
l_{M}^{3}=\pi l_{3} l^{2}
$$


Finally, it is natural in three dimensions to combine the dualized electric field $\tau_{1}$ and the Calabi-Yau volume $\tau_{2}$ into one complex scalar $\tau$ as:

$$
\tau=\tau_{1}+i \tau_{2} .
$$

One can then check that for an ansatz of the form specified above, the eleven-dimensional equations of motion become equivalent to those of the three-dimensional action (3.1). In such a reduction the sphere-wrapping M2-branes can be described as charged particles:

$$
\frac{S_{M 2}}{2 \pi}=-\frac{1}{l_{3}} \int d \xi \frac{\sqrt{-g}}{\tau_{2}}+\int A .
$$

We would like to point out a few interesting facts about this reduction:

- We were able to reduce assuming a constant radius for the $S^{2}$ because none of the other five-dimensional fields or the M2-brane source terms couple to the volume of the sphere. The latter is a general property of codimension-two branes: if one considers a codimension-two brane wrapped on a compact manifold, and reduces over this manifold, the coupling of the BI action to the volume modulus is balanced by the Weyl transformation needed to go to the lower-dimensional Einstein frame.

- Although we will be mainly interested in the M-theory origin of the three-dimensional system (3.1) discussed above, solutions to (3.1) can of course be embedded in any higher dimensional theory that allows (3.1) as a consistent truncation. Of special physical interest are embeddings in a type IIB string theory on $S^{3} \times \mathcal{M}_{4}$ (with $\mathcal{M}_{4}$ either $K_{3}$ or $T_{4}$ ), which is the near horizon limit of the D1-D5 system. In the probe approximation, branes wrapping the $S^{3}$ have been conjectured to account for the entropy of the Strominger-Vafa black hole in a similar manner as the $\mathrm{S}^{2}$-wrapping M2-branes in the M-theory frame did for the current setup [28]. Performing an Sduality, one obtains another interesting duality frame where only NS sector fields are excited and which could be the starting point for a sigma-model description. In another duality frame of interest, our system describes the backreaction of D7branes wrapped on $\mathrm{S}^{3} \times \mathcal{M}_{4}$. In this frame, the field $\tau$ is the standard axion-dilaton of type IIB. Our configurations can then be viewed as nontrivial solutions of FTheory. In Appendix A we discuss how our solutions can be embedded into different higher-dimensional theories by showing the explicit U-duality chain. An overview is presented in Table 1.

- Instead of compactifying to three dimensions, one can instead consider the fivedimensional theory obtained by reduction on the Calabi-Yau alone. This reduction gives us the action of $\mathcal{N}=1$ supergravity in five dimensions. The complex scalar $\tau$ is then part of the universal hypermultiplet. To account for the charge and backreaction of probe branes wrapped on $\mathrm{S}^{2}$ we must seek solutions with non-trivial hyperscalars turned on. There is an extensive literature on the general framework of finding solutions in this situation [29, 30, 31, 32], though few specific examples are known. Throughout the rest of the paper we will focus on the M-theory language 


\begin{tabular}{|c|c|c|}
\hline theory & background branes & source branes \\
\hline$M$ on $\mathrm{S}^{2} \times T^{6}$ & $M 5$ 's & $M 2$ on $\mathrm{S}^{2}$ \\
$I I B$ on $\mathrm{S}^{3} \times T^{4}$ & $D 3-D 3$ & $D 3$ on $\mathrm{S}^{3}$ \\
$I I B$ on $\mathrm{S}^{3} \times T^{4}$ & $D 1-D 5$ & $D 5$ on $\mathrm{S}^{3} \times T^{2}$ \\
$I I B$ on $\mathrm{S}^{3} \times T^{4}$ & $F 1-N S 5$ & $N S 5$ on $\mathrm{S}^{3} \times T^{2}$ \\
$F$ on $\mathrm{S}^{3} \times T^{6}$ & $D 3-D 3$ & $D 7$ on $\mathrm{S}^{3} \times T^{4}$ \\
\hline
\end{tabular}

Table 1: Embeddings of the three-dimensional axion-dilaton system in various higher dimensional theories related by U-duality. The axion-dilaton $\tau$ is in each case a modulus of the toroidal compactification. The background branes produce a near horizon $\mathrm{AdS}_{3}$ flux compactification, and the source branes couple to $\tau$.

and the three-dimensional action which are more straightforward. However, it is important to note that our general ansatz and solutions are non-trivial solutions of the five-dimensional theory. To the extent of the authors' knowledge these solutions are the first non-singular examples of such type. It might be interesting to make the connection to the geometric language of [29] more precise.

\section{Holomorphic BPS solutions}

In this section we study stationary solutions to the action (3.1) and display an ansatz that will lead to BPS solutions. Before we proceed with solving the equations of motion, we remark that we are looking at a special system. In three dimensions, the M2-branes wrapped on the sphere correspond to particles and have a one-dimensional worldvolume. However, charged codimension-two objects exhibit non-generic behaviour. Unlike many other (p-) brane solutions, the fields they source depend not only on one radial variable, but on the two directions of the transverse space. This was shown for the flat space analog of our system for instance in the case of cosmic strings in four dimensions [13] and later applied to seven-branes in type IIB supergravity [33]. Our work generalises such systems by the addition of a negative cosmological constant.

Ansatz: The equations of motion one obtains from (3.1) are

$$
\begin{array}{r}
R_{\alpha \beta}+\frac{2}{l^{2}} g_{\alpha \beta}=(\mu-1) \frac{\partial_{(\alpha} \tau \partial_{\beta)} \bar{\tau}}{\tau_{2}^{2}}, \\
\partial_{\alpha}\left(\sqrt{-g} g^{\alpha \beta} \partial_{\beta} \tau\right)+i \sqrt{-g} g^{\alpha \beta} \frac{\partial_{\alpha} \tau \partial_{\beta} \tau}{\tau_{2}}=0 .
\end{array}
$$

It is important to note that, due to the specific 'non-standard' kinetic term for the scalar $\tau$, equation (4.2) - from varying with respect to $\bar{\tau}$ - only features derivatives of $\tau$ and not of $\bar{\tau}$. Furthermore, since the probe branes we started from were time-independent for some specific timelike coordinate $t$ (see (2.2)), we seek stationary solutions: $\partial_{t} \tau=0$. As was shown in [13] for the flat space analog of our system $(l=\infty)$, these two facts combined imply that the scalar equation of motion can be solved by choosing $\tau$ to be (anti)-holomorphic in 
the complex coordinate naturally made up of the remaining two spatial coordinates. One can see from (4.2) that this remains true even if the full three-dimensional metric is not flat but when the spatial part of $\sqrt{g} g^{\alpha \beta}$ consists of constants.

Any stationary metric in three dimensions can be written as

$$
d s_{3 d}^{2}=\frac{l^{2}}{4}\left(-e^{2 \lambda}(d t+\chi)^{2}+e^{2 \phi} d s_{F}^{2}\right) .
$$

Here $d s_{F}^{2}$ is a flat metric on the two spatial directions, $\phi$ and $\lambda$ are functions of the spatial coordinates only and $\chi$ is a one-form on the spatial part.

The assumption that for the spatial directions $\sqrt{g} g^{\alpha \beta}=$ constant is equivalent to requiring $\lambda=0$. Choosing complex coordinates on $d s_{F}^{2}$, our metric ansatz takes the form

$$
d s_{3 d}^{2}=\frac{l^{2}}{4}\left(-(d t+\chi)^{2}+e^{2 \phi(z, \bar{z})} d z d \bar{z}\right) .
$$

There is still some gauge freedom left in this ansatz. We can make a conformal transformation $z \rightarrow g(z)$ which does not alter the form of the metric but sends

$$
\phi(z, \bar{z}) \rightarrow \phi(z, \bar{z})+\frac{1}{2} \log \partial g(z)+\frac{1}{2} \log \bar{\partial} \bar{g}(\bar{z}) .
$$

There is also a local shift symmetry

$$
t \rightarrow t-f(z, \bar{z}), \quad \chi \rightarrow \chi+d f .
$$

Equations of motion: With the metric ansatz (4.4), the $\tau$ equation of motion (4.2) reduces to

$$
\partial \bar{\partial} \tau+i \frac{\partial \tau \bar{\partial} \tau}{\tau_{2}}=0 .
$$

It can be solved by taking $\tau$ to be an arbitrary holomorphic or antiholomorphic function, and we will see in the next section that this leads to supersymmetric solutions.

Using our ansatz (4.4), the Einstein equations (4.1) can be written as

$$
\begin{aligned}
d \chi & =\frac{i e^{2 \phi} d z \wedge d \bar{z}}{2}, \\
\partial \bar{\partial} \phi-\frac{e^{2 \phi}}{4} & =-(\mu-1) \frac{\partial \tau \bar{\partial} \bar{\tau}}{4 \tau_{2}^{2}} .
\end{aligned}
$$

The equation for $\phi$ is the Liouville equation with a source term provided by $\tau$.

Before we solve these equations, it is instructive to note the differences with the flat space limit of [13]. In that flat space scenario, the equation for the conformal factor $\phi$ is a Poisson equation with source where here we found a sourced Liouville equation. Another important difference is the topology of the spatial base manifold. In the presence of a cosmological constant the spatial base manifold has the conformal structure and topology of the disk, as opposed to the Minkowski case where the topology and conformal structure underlying the equations is that of the Riemann sphere. In our case the equations (4.8),(4.9) still have an elegant solution, but it is not straightforward to construct 'stringy' solutions where $\tau$ has nontrivial $S L(2, \mathbb{Z})$ monodromies as was done in [13]. 


\section{Constant axion-dilaton: $\mathbf{A d S}_{3}$}

When $\tau$ is constant, we are in the pure gravity case and the metric (4.4) describes local $\mathrm{AdS}_{3}$, written as a timelike fibration over Euclidean $\mathrm{AdS}_{2}$. We illustrate how $\mathrm{AdS}_{3}$ can be written in this form, and introduce two coordinate systems that will appear later on.

The general solution to the Liouville equation (4.9) without source and to the equation for the one-form $\chi(4.8)$ is $^{2}$

$$
\begin{aligned}
e^{2 \phi} & =\frac{4 \partial g \bar{\partial} \bar{g}}{(1-g \bar{g})^{2}}, \\
\chi & =2 \operatorname{Im} \partial \phi+d f,
\end{aligned}
$$

where $g(z)$ is an arbitrary holomorphic function and $f(z, \bar{z})$ is an arbitrary real function. These arbitrary functions reflect the conformal invariance (4.5) and the shift symmetry (4.6) of our ansatz. The resulting metric is locally $\mathrm{AdS}_{3}$. To see this more explicitly, one can make the following coordinate transformation to global $\mathrm{AdS}_{3}$ coordinates $(\sigma, \rho, \psi)$ :

$$
\begin{aligned}
\sigma & =\frac{t+f}{2} \\
\rho & =\operatorname{arctanh}|g|, \\
\psi & =-\arg (g)+\frac{t+f}{2},
\end{aligned}
$$

in terms of which one obtains the standard global $\mathrm{AdS}_{3}$ metric (2.1). As discussed in Sec. 2 , the $\mathrm{AdS}_{3}$ vacuum preserves 8 Killing spinors in the theory under consideration. These were labelled as $g_{\frac{m}{2}}^{\alpha a}$ and correspond to the fermionic generators of $S U(1,1 \mid 2)_{L}$. They are computed in Appendix B and are given by

$$
g_{\frac{m}{2}}^{\alpha a}=\frac{\sqrt{l}}{\sqrt{2} \tau_{2}^{1 / 6}} e^{\frac{i m}{2}(t+f)} e^{-\frac{i \alpha \varphi}{2}} e^{\frac{i \theta}{2} \gamma^{\hat{\varphi}}} g_{0}^{\alpha a} \frac{m}{2},
$$

where the $g_{0}^{\alpha a} \frac{m}{2}$ are suitably chosen constant spinors given in (B.18).

Two coordinate systems will be of use later. In the first coordinate system, which we call 'disk coordinates' $(t, z, \bar{z})$, the spatial base is the Poincaré disk. We take $z, \bar{z}$ to range over the unit disk in the complex plane, $|z|<1$, and choose $g(z)=z, f=0$. Putting $z=r e^{i \varphi}$ we find

$$
d s^{2}=\frac{l^{2}}{4}\left[-\left(d t+\frac{2 r^{2}}{1-r^{2}} d \varphi\right)^{2}+\frac{4}{\left(1-r^{2}\right)^{2}}\left(d r^{2}+r^{2} d \varphi^{2}\right)\right] .
$$

This coordinate system covers $\mathrm{AdS}_{3}$ globally as one can check using (4.12). It is related to the coordinate system (2.2) in Sec. 2 by a redefinition of the radial coordinate $r=\tanh \rho$. The boundary of $\mathrm{AdS}_{3}$ is a cylinder formed by the unit circle and the time coordinate.

In another coordinate system, which we will call 'upper half plane coordinates' $(t, w, \bar{w})$, the base is the hyperbolic plane. We take coordinates $w, \bar{w}$ ranging over the upper half

\footnotetext{
${ }^{2}$ For notational simplicity we are a bit sloppy in distinguishing between the holomorphic partial derivative and the corresponding Dolbeault operator, denoting both with $\partial$. We trust the reader to distinguish between them by checking if the result is a scalar or differential form.
} 
plane, $\operatorname{Im} w>0$, and take $g(w)=(w-i) /(w+i), f=0$. Putting $w=x+i y$, the metric becomes

$$
d s^{2}=\frac{l^{2}}{4}\left[-\left(d t+\frac{d x}{y}\right)^{2}+\frac{d x^{2}+d y^{2}}{y^{2}}\right] .
$$

This is again a global coordinate system ${ }^{3}$, and the spatial boundary now consists of the real line and the point $w=i \infty$.

\section{Holomorphic axion-dilaton solutions: Gödel space}

We explore non-trivial solutions to the equations $(4.8,4.9)$ where the axion-dilaton field is nonconstant. We will construct supersymmetric solutions which should be seen as the backreacted geometries due to wrapped M2-brane sources. The class of solutions we find, has brane sources on the boundary and its local geometry is that of the three-dimensional Gödel universe. In fact, the simplest solution is global Gödel space, thus providing a new supersymmetric embedding of the Gödel universe in string/M-theory.

Solving the equations: We can solve the $\tau$ equation (4.7) by taking $\tau$ to be a holomorphic function:

$$
\tau=\tau(w) .
$$

As we will show below, this will lead to $1 / 2$-BPS solutions ${ }^{4}$.

Next we turn to the equation for the one-form $\chi$ (4.8). We see that it is solved by a simple modification of (4.11):

$$
\chi=2 \operatorname{Im}\left(\partial \phi+(1-\mu) \partial \ln \tau_{2}\right)+d f .
$$

Again, $f$ is an arbitrary real function reflecting the shift symmetry (4.6).

It remains to solve the Liouville equation (4.9) for the conformal factor $e^{2 \phi}$ in the presence of the source term. We write the equation as

$$
\mathcal{D} e^{2 \phi} \equiv\left(\partial \bar{\partial} \ln -\frac{1}{2}\right) e^{2 \phi}=-(\mu-1) \frac{\partial \tau \bar{\partial} \bar{\tau}}{2 \tau_{2}^{2}} .
$$

The source term on the right-hand side is quite special in that it is an eigenfunction of the the non-linear differential operator $\mathcal{D}=\partial \bar{\partial} \ln -\frac{1}{2}$. Indeed, we can write it as

$$
-(\mu-1) \frac{\partial \tau \bar{\partial} \bar{\tau}}{2 \tau_{2}^{2}}=\mathcal{D}\left(\mu \frac{\partial \tau \bar{\partial} \bar{\tau}}{\tau_{2}^{2}}\right)
$$

Therefore a solution to the equation is given by

$$
e^{2 \phi}=\mu \frac{\partial \tau \bar{\partial} \bar{\tau}}{\tau_{2}^{2}}
$$

\footnotetext{
${ }^{3}$ We should warn the reader that the $t$ coordinate in (4.15) differs from the one in (4.14) by a shift transformation: $t \rightarrow t+2 \arg (1-z)$.

${ }^{4}$ We could also take $\tau$ to be antiholomorphic, which would correspond to replacing brane sources with antibranes and would preserve different supersymmetries.
} 
This solution is a special case of those obtained in [34]. Let us discuss the uniqueness of our solution. As far as the authors know, there does not exist a proof of uniqueness of the solution (4.20) in the literature. Nevertheless, since we are working in three-dimensional gravity, we know that a given energy-momentum tensor completely determines the local geometry, so that other solutions to (4.9) (if any) must lead to a locally equivalent metric. Boundary conditions can then provide the global structure. However as we will see there seems to be a unique simply connected and geodesically complete solution. In this solution the spatial base, parameterized by $(w, \bar{w})$, has the conformal structure and topology of the disk, as in the case of global $\mathrm{AdS}_{3}$. In this paper we will not explicitly consider the interesting generalization of taking the base to be a quotient of this disk and $\tau$ to have nontrivial $S L(2, \mathbb{Z})$ monodromies. We hope to return to this in the future.

Supersymmetry properties: Let us discuss the supersymmetry properties of the solutions $(4.16,4.17,4.20)$ when embedded in five- or eleven-dimensional supergravity. The bosonic symmetry group is broken to $U(1)_{L} \times S U(2)_{L} \times S L(2, \mathbb{R})_{R}$. In Appendix B, we show that the backgrounds with nonconstant $\tau$ are supersymmetric and preserve half of the supersymmetries preserved in the constant $\tau$ case. The preserved Killing spinors are precisely of the form (4.13) (with $\tau_{2}$ no longer constant), subjected to an additional projection condition leaving one half of the supersymmetries preserved by $\mathrm{AdS}_{3}$. In terms of our basis (4.13) of the $S U(1,1 \mid 2)$ superalgebra, the Killing spinors preserved after turning on a holomorphic $\tau$ are ${ }^{5}$

$$
g_{\frac{1}{2}}^{\alpha+}, g_{-\frac{1}{2}}^{\alpha-} .
$$

This is in precise agreement with the analysis (2.5) in the probe approximation, and confirms our interpretation that our solutions represent backreacted wrapped M2-branes. As a further check on this interpretation, one can verify that placing a wrapped M2-brane probe in our nontrivial background does not break any further supersymmetries, as is expected from standard D-brane lore, by studying the $\kappa$-symmetry conditions.

The Gödel solution: As discussed above, we can take $\tau$ to be any single-valued (possibly multiple-to-one) meromorphic function from the upper half plane to itself. The simplest case, which we will study in the remainder of this work, is to take

$$
\tau(w)=w
$$

which is one-to-one and has a first order pole on the boundary at $w=i \infty$. More general multiple-to-one maps can be locally brought into this form by a conformal transformation. Choosing $f=0$ in (4.17) and defining $w=x+i y$ we obtain the metric

$$
d s^{2}=\frac{l^{2}}{4}\left[-\left(d t+\mu \frac{d x}{y}\right)^{2}+\mu \frac{d x^{2}+d y^{2}}{y^{2}}\right] .
$$

Rescaling $t \rightarrow \mu t$ one sees that this is the metric of timelike warped AdS (see e.g. [35]).

\footnotetext{
${ }^{5}$ If we had turned on antiholomorphic $\tau$, we would have preserved the other half of the supersymmetries, i.e. $g_{\frac{1}{2}}^{\alpha-}, g_{-\frac{1}{2}}^{\alpha+}$.
} 
For $\mu>1$, including the case of interest $\mu=3 / 2$, the timelike fiber is stretched, and the space is known to be the Gödel geometry [36]. Gödel's original solution [14] corresponds to setting $\mu=2$. This is one of the first examples of a three-dimensional supersymmetric Gödel space in the literature, see also [15].

Formally one could also take $\mu<1$, in which case the timelike fibre is squashed with respect to pure $\mathrm{AdS}_{3}$. This space has no closed timelike curves [35] and also appears as a solution to topologically massive gravity [37]. However it arises from an unphysical matter source: as we can see from (3.1), it requires a 'ghost' axion-dilaton with a wrong sign kinetic term. Alternatively, one can see it as coming from a perfect fluid source with negative energy density (see (4.24) below). We will restrict attention to $\mu>1$ in what follows.

Gödel's solution was originally obtained as a solution of gravity with negative cosmological constant $\Lambda=-1 / l^{2}$ in the presence of a pressureless fluid source. It is instructive to check that the energy-momentum tensor of our scalar field solution $\tau=x+i y$ behaves exactly as a pressureless fluid:

$$
\begin{aligned}
T_{\mu \nu} & \equiv-\frac{4 \pi}{l_{3} \sqrt{-g}} \frac{\delta S_{\tau}}{\delta g^{\mu \nu}} \\
& =\frac{4 \pi(\mu-1)}{l_{3} \tau_{2}^{2}}\left[\partial_{(\mu} \tau \partial_{\nu)} \bar{\tau}-\frac{1}{2} g_{\mu \nu} \partial_{\rho} \tau \partial^{\rho} \bar{\tau}\right] \\
& =\rho u_{\mu} u_{\nu},
\end{aligned}
$$

where the unit vector is $u^{\mu}=\frac{2}{l} \delta_{0}^{\mu}$ and the energy density of the fluid is

$$
\rho=\frac{16 \pi(\mu-1)}{\mu l_{3} l^{2}} .
$$

Setting $\mu=2$ we again find the expression in [14]. The fluid flow is rotational since $\star_{3}(u \wedge d u)$ is a nonzero constant, indicating that Gödel space rotates around every point.

It is well-known that Gödel space suffers from causal pathologies in the form of closed timelike curves. These are most apparent in the coordinate system which has the Poincaré disk as the spatial base. The following coordinate transformation takes us to this frame:

$$
\begin{aligned}
t & \rightarrow t+2 \mu \arg (1-z), \\
w & \rightarrow i \frac{1+z}{1-z}
\end{aligned}
$$

We define $z=r e^{i \varphi}$ to get

$$
d s^{2}=\frac{l^{2}}{4}\left[-\left(d t+\mu \frac{2 r^{2}}{1-r^{2}} d \varphi\right)^{2}+4 \mu \frac{d r^{2}+r^{2} d \varphi^{2}}{\left(1-r^{2}\right)^{2}}\right] .
$$

In the above form of the metric, it is easy to see that the vector field $\partial_{\varphi}$ becomes timelike for $r>\frac{1}{\sqrt{\mu}}$, so that $\varphi$-circles become closed timelike curves for these values of the radius. The disk coordinate frame is also useful to visualize the axion-dilaton solution. The brane source is located at the point $z=1$ on the boundary. The lines of constant dilaton $\tau_{2}$ are circles tangent to $z=1$. Using (3.6),(4.22) one can show that $\tau_{2}$ also plays the role 
of the scalar potential for the electric field, so these circles are also equipotential surfaces. The electric field lines are the lines of constant $\tau_{1}$ and are orthogonal to the equipotential circles. These properties are illustrated in Fig. 2.

The source: Since $\tau$ has a pole at infinity in the upper half plane coordinates, we expect to have a brane-like source of the form (3.9) there. This was also nicely apparent in Fig. 2, where we plotted the electric fieldlines. Let us compute the total charge of this source. It's convenient to make an $S L(2, \mathbb{Z})$ transformation and consider

$$
\tau=-1 / w,
$$

so that the location of the pole is now at the origin. As $\tau_{1}$ is related to the $U(1)$ field sourced by M2-branes we expect the source to be made up of these. To be more precise, the elevendimensional definition of M2-brane charge is:

$$
q_{M 2}=\int_{\Sigma_{7}} \star_{11} F_{4} .
$$

Using the compactification ansatz (3.4) we can rewrite this definition in three-dimensional form:

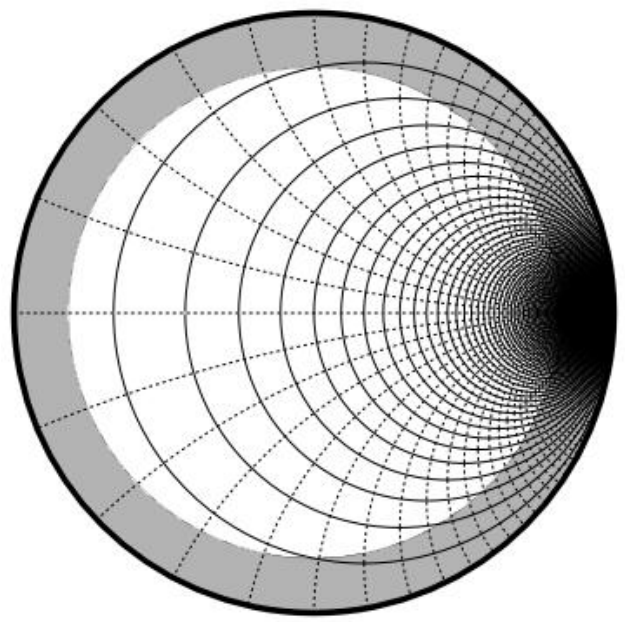

Figure 2: The Gödel solution in disk coordinates. Circles within the gray zone and centered at the origin are closed timelike curves. The solid lines are equipotential surfaces (constant $\tau_{2}$ ), the dotted lines are electric field lines (constant $\tau_{1}$ ). The brane source is at $z=1$.

$$
q_{M 2}=\int_{\gamma} d \tau_{1},
$$

here $\gamma$ is a curve in the spatial base of the three-dimensional metric. Plugging in the explicit form (4.28) of $\tau_{1}$ for the Gödel background and taking the limit where $\gamma$ becomes the real line one finds that

$$
q_{M 2}=\int_{-\infty}^{\infty} \frac{d x}{x^{2}}=+\infty
$$

Hence we should see the pole in $\tau$ as coming from a source of infinite charge. The same conclusion is of course reached by computing the source for the dilaton field. The fact that the global Gödel space carries an infinite amount of charge is probably due to the fact that the axion-dilaton solution covers an infinite number of fundamental $\operatorname{SL}(2, \mathbb{Z})$ domains. It seems plausible that one can obtain finite charge solutions by taking appropriate quotients of the global Gödel space.

\section{Joining Gödel to AdS}

One of our original motivations in studying the system (3.1) was to analyse solutions corresponding to branes wrapped around the $\mathrm{S}^{2}$ of an $\mathrm{AdS}_{3} \times \mathrm{S}^{2}$ geometry. Since such states carry a non-trivial topological charge that appears as a central element of the supersymmetry algebra it was to be expected that such solutions are no longer asymptotically AdS. 
However, both from the point of view of holography and from the black hole microstate motivation it would be interesting if there was some kind of 'embedding' of these solutions into an asymptotic AdS spacetime. Probably the most straightforward way of realising such a setup is by enclosing a Gödel region carrying the M2-charge by a domainwall that cancels this charge. Then, as in three dimensions all vacuum spacetimes are locally $\mathrm{AdS}_{3}$, on the other side of the wall we are guaranteed to find a local $\mathrm{AdS}_{3}$ spacetime. In this section we will realise exactly this idea, although it turns out that, under our assumptions, demanding that the AdS-side of the domainwall is connected to the boundary is equivalent to having a negative tension domainwall. In the case we have the Gödel part of spacetime on the outside then the domainwall is made up of more familiar positive tension, smeared out M2-branes. For an overview see Fig. 3.

Another motivation to consider such a domainwall construction is the analogy to [38, $39,18]$. In these references the authors show that one can remove the closed timelike curves of Gödel-like spacetimes by introducing a domainwall that connects it to an AdSlike spacetime. Naively one would hope the same effect to take place in the current setup. However, using the specific domainwall ansatz below, this seems not to be the case, as closed timelike curves are present in the spacetime even after introducing the domainwall. The authors were not able to find a more general construction that eliminates the closed timelike curves. This however remains an interesting goal for future research. It would also be interesting to see if there are smooth interpolating solutions in the spirit of $[19,20,40]$, describing these domainwall configurations.

The domainwall: More concretely, when trying to connect, or 'glue', the Gödel space through a domainwall to $\mathrm{AdS}_{3}$, it is clear that we need to cancel M2-charge on one side of the wall, and hence the domainwall should carry this charge. It thus seems natural to assume that the domainwall is made up of M2-branes that wrap the internal $\mathrm{S}^{2}$. Since these branes are point particles in the three non-compact dimensions we need to smear them along a spacelike direction to make a domainwall ${ }^{6}$ out of them. By these assumptions we extend our previous Lagrangian (3.1) with a domainwall source made up of M2-particles (3.9):

$$
\begin{aligned}
\frac{l_{3} L}{2 \pi}= & \sqrt{-g}\left(R+\frac{2}{l^{2}}-(\mu-1) \frac{\partial_{\mu} \tau_{2} \partial^{\mu} \bar{\tau}_{2}}{\tau_{2}^{2}}-\frac{(\mu-1) \tau_{2}^{2}}{2} F_{\mu \nu} F^{\mu \nu}\right) \\
& -2 \alpha(\mu-1) \int d \lambda d \sigma \delta^{3}\left(x^{\mu}-X^{\mu}(\sigma, \lambda)\right)\left(\frac{\sqrt{-g_{\mu \nu}(X) \partial_{\sigma} X^{\mu} \partial_{\sigma} X^{\nu}}}{\tau_{2}(X)}-\beta \frac{A_{\mu}(X)}{2} \partial_{\sigma} X^{\mu}\right)
\end{aligned}
$$

Note that here we found it more convenient to replace the real scalar $\tau_{1}$ with its Hodge dual $F=d A$ as in (3.6), $\sigma$ parametrizes the M2-worldline and $\lambda$ is a smearing parameter.

For the moment we will leave the tension $\alpha$ arbitrary and take $\beta= \pm 1$. Furthermore, we introduced the parameter $\mu$ also in the domainwall action as three-dimensional solutions for abritary values of $\mu$ might be of independent interest. As before, the main value of

\footnotetext{
${ }^{6}$ As the smearing does not induce tension along that direction some might rather prefer to call such a configuration a charged dust shell instead of a domainwall.
} 


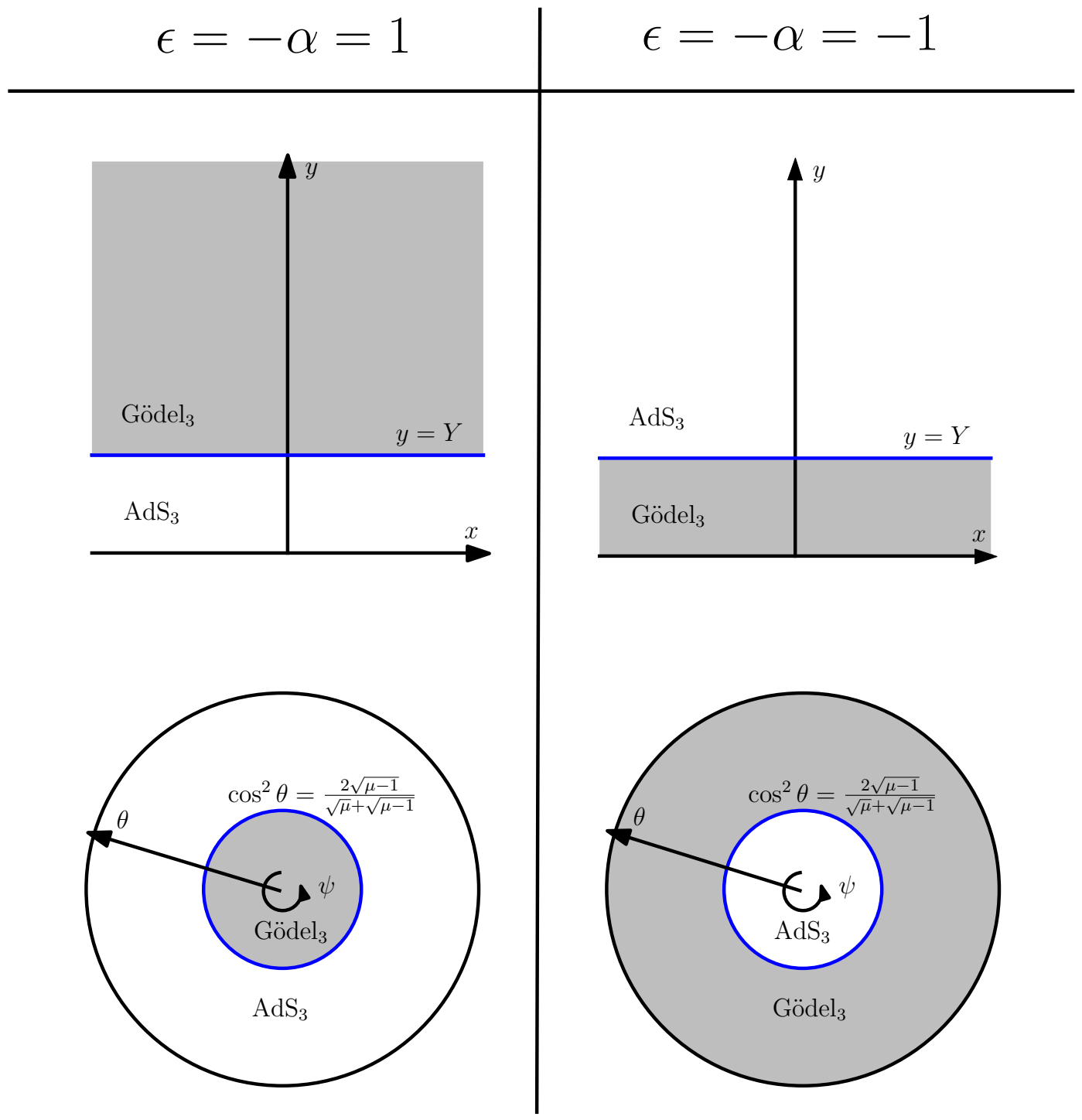

Figure 3: This figure gives an overview of the different gluings. On top we have presented the picture in the upper half plane coordinates $x, y$, most natural to Gödel space at $t=\mathrm{cst}$. As discussed in the main text, in these coordinates one still has to make an identification. On the bottom the glued spacetime is presented in global AdS coordinates. The disk shown here is a $\sigma=$ cst slice of the cylinder that is $\mathrm{AdS}_{3}$. Note that we have introduced the standard Penrose coordinate $\theta$, defined by $\tan \theta=\sinh \rho$. The left hand side is that for the choice of $\epsilon=1$ and $\alpha=-1$. In this case the AdS part of space connects to the boundary, but the domainwall has negative tension. On the right hand side the situation is depicted for the opposite choice. Now the Gödel part connects to the boundary and the domainwall has positive tension and an interpretation as smeared M2-particles.

interest is $\mu=3 / 2$, which is the only value that corresponds to the M-theory embedding as given in section 3 . In that case (and when $\alpha>0$ ), the domainwall can be interpreted as smeared M2-branes (or anti M2 branes depending on the sign of $\beta$ ).

Since the domainwall source couples to the dilaton $\tau_{2}$, which in the Gödel solution is given by $\tau_{2}=y$, one can only smear with a constant density along a surface of constant 
$y$. For the rest of this section we will be working in the specific coordinates $\left(x^{0}, x^{1}, x^{2}\right)=$ $(t, x, y)$, known from the Gödel solution (4.22), and assume the following embedding of the domainwall:

$$
X^{0}(\sigma, \lambda)=\sigma, \quad X^{1}(\sigma, \lambda)=\lambda, \quad X^{2}(\sigma, \lambda)=Y .
$$

Furthermore, we will assume all fields to be continuous in $y$ in spacetime, although we will allow derivatives of the fields to be discontinuous at $y=Y$. This can be achieved by 'gluing' two different solutions of the Lagrangian (3.1) ((5.1)) without domainwall source) together at $y=Y$. More concretely we propose the following field configuration:

$$
\begin{aligned}
d s^{2} & =N^{2} d y^{2}+h_{a b} d x^{a} d x^{b}, \\
N & =N_{\text {Gödel }} \Theta[\epsilon(y-Y)]+N_{\operatorname{AdS}} \Theta[\epsilon(Y-y)], \\
h_{a b} & =h_{a b}^{\text {Gödel }} \Theta[\epsilon(y-Y)]+h_{a b}^{\operatorname{AdS}} \Theta[\epsilon(Y-y)], \\
A & =\left(\frac{l}{y} \Theta[\epsilon(y-Y)]+\frac{l}{Y} \Theta[\epsilon(Y-y)]\right) d t+\left(\frac{l \mu}{2 y^{2}} \Theta[\epsilon(y-Y)]+\frac{l \mu}{2 Y^{2}} \Theta[\epsilon(Y-y)]\right) d x, \\
\tau_{2} & =y \Theta[\epsilon(y-Y)]+Y \Theta[\epsilon(Y-y)] .
\end{aligned}
$$

Here the Heaviside stepfunction, $\Theta(x)$, gives a formal way of defining the gluing. We introduced a sign $\epsilon= \pm 1$ to distinguish between the solution with AdS/Gödel 'inside' or 'outside'. I.e. when $\epsilon=1$ we have Gödel at $y>Y$ (inside) and AdS at $y<Y$ (outside), when $\epsilon=-1$ the situation is exactly opposite. Note that on the AdS side, we take the complex scalar $\tau$ (written here as $\tau_{2}$ and a gauge field $A$ ) to be constant. As we will show below, the chosen values of these fields make sure the ansatz for $A$ and $\tau_{2}$ is valid and satisfies the corresponding equations of motion. Note that in the above ansatz the only remaining unknowns are $N_{\mathrm{AdS}}$ and $h_{a b}^{\mathrm{AdS}}$. Their Gödel counterparts are known by definition and can be read off from (4.22). Although we know on general grounds that the metric on the other side will be AdS, we do not know the precise form of the functions $N$ and $h_{a b}$ on that side, as $t, x$ and $y$ are an unknown set of coordinates there. As we will explicitly work out below, the precise form of these functions can be found by solving the Einstein equations with suitable boundary conditions. Of course, by definition of our gluing procedure, we want the total metric to be continuous, so this imposes the condition:

$$
\begin{aligned}
& \left.N_{\text {AdS }}\right|_{y=Y}=\left.N_{\text {Godel }}\right|_{y=Y}=\frac{l^{2}}{4 Y^{2}}, \\
& \left.h_{a b}^{\mathrm{AdS}} d x^{a} d x^{b}\right|_{y=Y}=\left.h_{a b}^{\text {Godel }} d x^{a} d x^{b}\right|_{y=Y}=-\frac{l^{2}}{4}\left(d t^{2}+\frac{2 \mu d x d t}{Y}+\frac{\mu(\mu-1)}{Y^{2}} d x^{2}\right) .
\end{aligned}
$$

The junction conditions: We analyse the equations of motion following from (5.1) for the ansatz (5.3). Each of these equations can be split into three separate ones that have to be obeyed in the different regions: two bulk equations evaluated at $y>Y$ and $y<Y$ respectively and one 'singular' equation at $y=Y$. The bulk equations are the equations of motion following from the Lagrangian (3.1) without the domainwall source. Our ansatz automatically satisfies the bulk equation in the Gödel region, while in the AdS region $N_{\text {AdS }}$ and $h_{a b}^{\text {AdS }}$ have to be chosen so as to satisfy the vacuum Einstein equations. The 
singular equation at $y=Y$ is proportional to $\delta(y-Y)$ and receives two contributions: the first comes from the domainwall term in the Lagrangian, the second from possible singular derivatives of the fields at $y=Y$. In general relativity these singular equations that impose the equality between the domainwall source terms and the discontinuities of field derivatives are known as the Israel junction conditions [41]. In Appendix D we review the derivation of these equations and extend them to more general field theories. Note that the ansatz for the gauge field $A$ and scalar $\tau_{2}$ in (5.3) was chosen in such a way that continuity across $y=Y$ is assured.

As mentioned above, only the metric is not completely determined yet in our ansatz. Before deriving the correct form of the metric by the Israel junction conditions let us first check that the ansätze for the other fields satisfy their corresponding junction conditions. Applying the general formula (D.17) to the Lagrangian (5.1) and the fields $\tau_{2}$ and $A$ we get the following two equations (all evaluated at $y=Y$ ):

$$
\begin{aligned}
\frac{\Delta \partial_{y} \tau_{2}}{N} & =-\alpha \frac{\sqrt{-h_{00}}}{\sqrt{-h}} \\
N \sqrt{-h} \tau_{2}^{2} \Delta F^{y \mu} & =-\alpha \beta \delta_{t}^{\mu} .
\end{aligned}
$$

The definition of the operator $\Delta$ can be found in (D.3). Plugging in our gluing ansatz (5.3) these become

$$
\begin{aligned}
& \epsilon=-\alpha \\
& \beta=1 .
\end{aligned}
$$

So we see that by choosing the correct tension and charge the ansatz can be a solution for both signs of $\epsilon$. Note however, that demanding the part of spacetime connected to the boundary at $y=0$ to be AdS, corresponds to $\epsilon=1=-\alpha$ and hence implies that the domainwall is made up of particles with negative tension.

Another equation that needs to be checked is that for the embedding scalars $X^{\mu}$. Based on the assumption of constant smearing density we made the ansatz (5.2) for these scalars, but we still have to verify if this is consistent with the equations of motion obtained by varying the Lagrangian (5.1) with respect to the $X^{\mu}$. This equation can be seen as a special case of a junction equation, but with only a contribution from the domainwall and no singular bulk terms. Plugging the ansatz (5.2) and the values of the other fields into those equations of motion leads directly to the condition

$$
\beta=1 \text {. }
$$

This is nicely consistent with the value of $\beta$ found above.

What remains is to solve the bulk Einstein equations on the AdS side with boundary conditions specified by the Israel matching conditions. Applying the Israel junction conditions (D.15) to the case at hand we find

$$
\Delta\left(K^{a b}-g^{a b} K\right)=\frac{-\alpha}{2 \tau_{2} N \sqrt{-h_{00}}} \delta_{t}^{a} \delta_{t}^{b} .
$$

These equations together with (5.4) should be interpreted to provide boundary conditions at $y=Y$ for the local AdS metric appearing in our ansatz (5.3). 
The Gödel-AdS solution: Solving the vacuum Einstein equations with the boundary conditions (5.10) and (5.4) at $y=Y$ is rather straightforward. One can verify that the solution for $\epsilon(Y-y)>0$ is given by

$$
d s^{2}=\frac{l^{2}}{4}\left(-\left(d t+\mu \frac{d x}{y}\right)^{2}+\frac{\mu}{y^{2}}\left(f(y) d x^{2}+f^{-1}(y) d y^{2}\right)\right)
$$

with

$$
f(y)=\mu+(1-\mu) \frac{y^{2}}{Y^{2}} .
$$

Although not the most familiar form, one can check that this metric is locally $\mathrm{AdS}_{3}$ by calculating the Ricci tensor. Furthermore it can be put in the standard global AdS coordinates $(2.1)$ by a simple coordinate transformation. Define the quantity $\omega=\frac{\sqrt{\mu(\mu-1)}}{Y}$. The transformation is then given by

$$
\begin{aligned}
\cosh 2 \rho & =\frac{\mu}{\omega y}, \\
\sigma & =\frac{\omega x+t}{2}, \\
\psi & =\frac{\omega x-t}{2} .
\end{aligned}
$$

This coordinate transformation shows that the domainwall at $y=Y$ maps to a cylindrical hypersurface at constant radius $\rho_{\star}$ in global $\mathrm{AdS}_{3}$, with $\cosh 2 \rho_{\star}=\sqrt{\frac{\mu}{\mu-1}}$. Note that in these coordinates the position of the domainwall is independent of the choice of $Y$. This is due to the fact that one could have removed $Y$ from the previous discussion by the transformation $x \rightarrow Y x, y \rightarrow Y y$, which is a symmetry of Gödel-space. We give an overview of the different gluings in the different coordinate systems in figure Fig. 3

As one knows from the standard coordinates on $\mathrm{AdS}_{3}$, the coordinate $\psi$ is periodic: $\psi \sim \psi+2 \pi \nu$. The most familiar value is $\nu=1$, which corresponds to global AdS. However one can also choose $\nu<1$, in which case the geometry corresponds to a conical defect geometry. Continuity requires that we impose the same periodicity on Gödel side as well; this leads to the identification

$$
\begin{aligned}
& t \sim t-2 \pi \nu, \\
& x \sim x+\frac{2 \pi}{\omega} \nu .
\end{aligned}
$$

It is however straightforward to check that this identification leads to new CTCs in the Gödel part of the glued spacetime. This holds in both cases of gluing, the one with Gödel at small $y(\epsilon=-1)$ and that at large $y(\epsilon=1)$. The reason is, that the Killing vector $\frac{1}{\omega} \partial_{x}-\partial_{t}$, that generates the identification, is timelike in Gödel both for $y \rightarrow 0$ and $y \rightarrow \infty .^{7}$

Let us also discuss the preservation of supersymmetry in our glued solutions. Since we need to impose the extra identification (5.16) on the Gödel part, Killing spinors should

\footnotetext{
${ }^{7}$ Note that one could generalise the identification in $\mathrm{AdS}$ to $(\psi, \sigma) \sim\left(\psi+2 \pi \nu_{1}, \sigma+2 \pi \nu_{2}\right)$. The metric would in this case become a spinning conical geometry, but it is easy to check that such an identification leads in general to closed timelike curves in both the Gödel part and the AdS part of the glued spacetime.
} 
be well-defined (i.e. either periodic or antiperiodic) under this identification. In the case $\nu=1$, we have global $\mathrm{AdS}_{3}$ on one side, preserving 8 supersymmetries. On the Gödel side, all 4 Killing spinors $(2.5,4.13)$ are antiperiodic under the identifications and are therefore globally well-defined. When $\nu<1$, the Killing spinors in the $\mathrm{AdS}_{3}$ part will pick up a complex phase under transport around the conical defect. As is well-known [42], in order to have globally well-defined Killing spinors one must compensate for this by turning on a Wilson line for one of the three-dimensional gauge fields that arise from the reduction of the five-dimensional metric on the two-sphere. From the five-dimensional point of view, this is nothing but a 'large' diffeomorphism of the form $\varphi \rightarrow \varphi \pm t$, where $\varphi$ is the angular coordinate on the two sphere. After performing this diffeomorphism, one can see that 4 out of 8 local supersymmetries exist globally on the AdS side. Doing the same diffeomorphism on the Gödel side preserves 2 out of 4 local Killing spinors, as one can see from (4.13). So we conclude that our glued geometries are supersymmetric, with the Gödel part preserving half of the supersymmetries of the AdS part in each case.

The case we would be most interested in is that of positive $\epsilon$, as then the glued spacetime is asymptotically AdS and we know how to do holography on such spaces. However, as can be read from the condition (5.7) and the Lagrangian (5.1), $\epsilon=1$ implies negative tension for the domainwall. Even though negative tension domain walls are not unheard of, either in supersymmetric theories (see e.g. [43]), or as orientifold-type objects in string/M theory (see e.g. [44, 45]), clearly it is harder to interpret them in terms of fundamental M-theory branes. It might still be interesting to understand these glued spaces in more detail through holography. Also, there is a potential danger of instabilities as discussed in [46], however since our construction preserves supersymmetry on both sides of the wall we believe this is not an issue in our case.

\section{Discussion and future directions}

In this work, we have constructed supersymmetric solutions to three-dimensional axiondilaton gravity with negative cosmological constant which describe the backreaction of $\mathrm{S}^{2}$-wrapped M2-branes in M-theory. We found a class of solutions where the axion-dilaton is (anti-) holomorphic and where the local geometry is that of the three-dimensional Gödel universe. We showed that these solutions preserve four supersymmetries in agreement with the analysis in the probe approximation. We have also shown that our solutions can be glued, in a supersymmetric manner, into asymptotically $\mathrm{AdS}_{3}$ geometries by including a charged domainwall.

Let us comment on some aspects which deserve a better understanding and some interesting directions for future research. A first puzzle is that our backreacted solutions have M2-brane sources only on the boundary, whereas in the probe approximation discussed in Sec. 2, it appeared as if the M2-branes could be placed anywhere. This could point to the existence of more general solutions with sources in the interior, but it could also be due to the fact that these are codimension-two objects producing long-range fields; hence the probe picture might be unreliable. Another feature of our solutions is that the brane charge residing on the boundary is actually infinite. This can be seen as a consequence of the fact 
that $\tau$ takes values in the entire upper half plane. One way to obtain a 'stringy' finite charge solution, would be to identify values of $\tau$ related by $S L(2, \mathbb{Z})$ transformations and make a similar identification on the coordinate $w$ of the base manifold. The charge integral (4.29) would then be finite. A similar procedure would work for an arithmetic subgroup of $S L(2, \mathbb{Z})$. One should note that such constructions in general involve identifications generated by timelike vectors and will produce more closed timelike curves. Nevertheless, such configurations are finite-energy, finite charge BPS solutions, and one would expect them to contribute to the path integral. It would be interesting to understand their role better.

Our original motivation for studying this system was the black hole microstate or deconstruction proposal $[6,7,8,9]$, where it was argued that $\mathrm{S}^{2}$ wrapped M2-brane probes have a large quantum mechanical degeneracy (coming from lowest Landau levels on the internal Calabi-Yau) that can account for the black hole entropy. An interesting question is whether this degeneracy can also be understood after including the backreaction. This might furthermore clarify the relation between these deconstruction states and other BPS solutions carrying the black hole charges that are more closely related to the original fuzzball proposal $[47,48,49]$. Although various BPS solutions were explicitly constructed, see e.g. $[50,51,52,53,54,19,20,40,55]$, it was argued recently that these might only account for a subleading fraction of the black hole ensemble $[56,57]$. It would in particular be interesting to understand the deconstruction microstates in a dual CFT and see if and how they evade the bound of [57]. Our gluing procedure in Sec. 5 should be seen as a first attempt in this direction. It would be interesting to explore more general gluings into $\mathrm{AdS}_{3}$ and the holographic interpretation of these constructions. Another approach to understand the quantum properties of the system (3.1) would be to argue the existence of a dual CFT in the spirit of the Kerr-CFT correspondence [58]. It is expected that, under suitable boundary conditions, the $U(1)_{L} \times S L(2, \mathbb{R})_{R}$ symmetry of the Gödel solution will extend to an asymptotic Virasoro algebra (see [59] for an example involving a Gödel solution with a different matter content). Since our system is supersymmetric for $\mu=3 / 2$, one would expect the asymptotic algebra to extend to a superconformal algebra in that case. It would also be interesting to see if the theory has interesting excitations, such as black hole solutions, which could be understood in the dual CFT.

\section{Acknowledgements}

The authors are especially grateful to E. Gimon and T. Wyder for collaboration on part of this work. During this work we benefitted from many discussions. It is a pleasure to thank D. Anninos, G. Compere, R. de Mello Koch, F. Denef, S. Detournay, S. El-Showk, T. Erler, B. Gaasbeek, M. Guica, M. Kleban, J. Manschot, G. Moore, P. Smyth and A. Van Proeyen. TSL was supported in part by the Natural Sciences and Engineering Research Council of Canada and the Institute of Particle Physics. JR was supported under a grant of the National Research Foundation of South Africa. His research has been supported in part by the EURYI grant EYI/07/E010 from EUROHORC and ESF. The work of DVdB is supported by the DOE under grant DE-FG02-96ER40949. The work of WVH and BV 
is supported in part by the FWO - Vlaanderen, project G.0235.05 and in part by the Federal Office for Scientific, Technical and Cultural Affairs through the Interuniversity Attraction Poles Programme Belgian Science Policy P6/11-P. B.V. is Aspirant of the FWO Vlaanderen and thanks the High Energy Theory Group at Harvard University for its hospitality.

\section{A. Other embeddings of the $3 \mathrm{D}$ system}

The three-dimensional theory (3.1) can arise as a consistent truncation of other theories than the M-theory context discussed in the main text. Here, we will discuss a few interesting alternative embeddings in type IIB string theory compactified on $\mathrm{S}^{3} \times \mathcal{M}_{4}$ where $\mathcal{M}_{4}$ is a compact 4-manifold. Such backgrounds arise for example in the near-horizon limit of the D1-D5 system and are of relevance in the description of the five-dimensional StromingerVafa black hole [60]. These alternative embeddings will be derived by applying U-dualities and the 4D-5D connection (see $[28,61]$ for more details on this procedure). We will first discuss the effect of these transformations on the 'background branes' that have an $\mathrm{AdS}_{3}$ near-horizon region, and then discuss the transformation of the 'source' branes that couple to the three-dimensional axion-dilaton and whose backreaction turns $\mathrm{AdS}_{3}$ into Gödel space.

We start with our M-theory embedding, replacing the Calabi-Yau manifold by $S_{M} \times$ $S_{5} \times \mathcal{M}_{4}$, where $S_{M}$ and $S_{5}$ are circles. For simplicity we take $\mathcal{M}_{4}$ to be a four torus, but most of the discussion applies to the case of $K_{3}$ as well. In particular, we take $\mathcal{M}_{4}=T_{2} \times T_{3}$ where $T_{2}, T_{3}$ are two-tori. For the background branes, we take M5-branes wrapping internal 4-cycles, extending along a common direction to form a BPS black string in five dimensions. For simplicity, we restrict to three types of M5-brane charges: we take $p^{1}$ M5-branes wrapping $T_{2} \times T_{3}$ and $p^{a}, a=2,3 \mathrm{M} 5$-branes wrapping $S_{M} \times S_{5} \times T_{a}$. The near-horizon geometry is an $\mathrm{AdS}_{3} \times \mathrm{S}^{2} \times S_{M} \times S_{5} \times \mathcal{M}_{4}$ background of the form (3.4) with constant $\tau$.

We perform the limits and dualities summarized in Table 2: First, we compactify on $S_{M}$ to type IIA: $p^{1}$ are now NS5-branes and the $p^{a}$ become D4-branes. Then we T-dualize on $S_{5}$ to get type IIB on the dual circle $\tilde{S}_{5} \cdot p^{1}$ becomes a KK monopole charge and $p^{a}$ are now D3-branes. Because of the presence of KK monopole charge, we can apply the standard 4D-5D connection [62] and obtain a five-dimensional configuration by taking the asymptotic size of $\tilde{S}_{5}$ to infinity. We then have a configuration of intersecting D3-branes forming a black string in a six-dimensional compactification of type IIB on $\mathcal{M}_{4}$. The nearhorizon geometry is $\operatorname{AdS}_{3} \times S^{3} / p^{1} \times \mathcal{M}_{4}$. By taking $p^{1}=1$, we get precisely $\operatorname{AdS}_{3} \times S^{3} \times \mathcal{M}_{4}$.

Let us follow what happens to the source branes under the above series of dualities. Starting from an M2-brane wrapping the $\mathrm{S}^{2}$ in the M-theory frame, one obtains a D3-brane wrapping the $\mathrm{S}^{3}$ in the type IIB frame. Hence in this frame, all the branes in the system are D3's! Starting from this configuration, one obtains some useful embeddings by applying further dualities. The results are summarized in Table 1 in the main text.

First, let's do two further T-dualities on $T_{2}$. The background branes are now the wellknown D1-D5 system. The source branes have become D5 branes wrapping $\mathrm{S}^{3} \times T_{2}$. In the probe picture, such branes were argued to have a high lowest Landau level degeneracy 


\begin{tabular}{|c|c|c|c|c|c|c|}
\hline $\mathrm{M} / S_{M} \times S_{5} \times \mathcal{M}_{4}$ & $\begin{aligned} \longrightarrow & \\
S_{M} & \rightarrow 0\end{aligned}$ & $\mathrm{IIA} / S_{5} \times \mathcal{M}_{4}$ & $\overrightarrow{\mathrm{T}\left(S_{5}\right)}$ & $\mathrm{IIB} / \tilde{S}_{5} \times \mathcal{M}_{4}$ & $\begin{array}{r}\tilde{S}_{5} \rightarrow \infty \\
\longrightarrow\end{array}$ & $\mathrm{IIB} / \mathcal{M}_{4}$ \\
\hline $\begin{array}{l}p^{1}: \mathrm{M} 5 / \mathcal{M}_{4} \\
p^{a}: \mathrm{M} 5 / S_{M} \times S_{5} \times T_{a}\end{array}$ & $\longrightarrow$ & $\begin{array}{l}\text { NS5 } / \mathcal{M}_{4} \\
\mathrm{D} 4 / S_{5} \times T_{a}\end{array}$ & $\longrightarrow$ & $\begin{array}{l}\text { KK5 } / \mathcal{M}_{4} \\
\text { D3 } / T_{a}\end{array}$ & $\longrightarrow$ & $\begin{array}{l}\text { (def. angle) } \\
\text { D3/Ta }\end{array}$ \\
\hline
\end{tabular}

Table 2: Dualities and 4D-5D connection

and account for the entropy of the five-dimensional Strominger-Vafa black hole [28]. If we perform a further S-duality, the background consists of fundamental strings and NS5branes, while the source branes are NS5-branes on $S^{3} \times T_{2}$, coupling to the complexified Kähler modulus of $T_{3}$. This frame is interesting because all the excitations are in the NS-sector, allowing for a sigma-model description of the system.

One can also obtain an F-theory embedding by starting from the intersecting D3-brane description and doing four T-dualities along $T_{2} \times T_{3}$. This just interchanges the two types of background D3-branes, while the source branes now become D7-branes wrapping $\mathrm{S}^{3} \times \mathcal{M}_{4}$. In this frame, $\tau$ is the standard axion-dilaton of type IIB, and the resulting solutions can be seen as nontrivial F-theory backgrounds involving D7-branes. It would be interesting to study the geometry of the fibration of the F-theory torus over Gödel space described by the $\tau$ field in more detail.

\section{B. Details of the supersymmetry analysis}

In this appendix we discuss the supersymmetry properties of our solutions. We will mostly use the eleven-dimensional supergravity point of view and comment on the analysis in five-dimensional $\mathcal{N}=1$ supergravity in Appendix $\mathrm{C}$.

\section{Conventions}

We will work in the eleven-dimensional supergravity conventions of [63]. The bosonic part of the eleven-dimensional supergravity (M-theory) action was given in (3.2). The Killing spinor equation is

$$
\nabla_{M} \epsilon+\frac{l_{M}^{3}}{12}\left[\Gamma_{M} \mathscr{H}_{4}-3 H_{M}\right] \epsilon=0,
$$

where $\not{f}=\frac{1}{4 !} F_{M N P Q} \Gamma^{M N P Q}, \oiint_{M}=\frac{1}{3 !} F_{M N P Q} \Gamma^{N P Q}$ and $\epsilon$ is an eleven-dimensional spinor satisfying the Majorana condition

$$
\epsilon^{*}=B \epsilon
$$

with $B$ a matrix satisfying $B \Gamma^{M} B^{-1}=\Gamma^{M *}$ which we will specify later.

We will consider bosonic field configurations of the form (3.4) with a three-dimensional metric of the form (4.3). To further simplify expressions, we will also use the explicit form of our solutions (4.16),(4.17),(4.20). We will show that all such configurations are supersymmetric solutions of eleven-dimensional supergravity. When $\tau$ is constant, the geometry is locally $\mathrm{AdS}_{3} \times \mathrm{S}^{2} \times C Y_{3}$ and the solution preserves 8 local Killing spinors. When $\tau$ is not constant, $\mathrm{AdS}_{3}$ is replaced by a local Gödel geometry and the number of local Killing spinors is 4 . 
It will be useful to work in a spinor basis adapted to the decomposition $S O(1,10) \supset$ $S O(1,4) \times S O(6)$. We decompose the eleven-dimensional Clifford matrices as (hatted indices refer to an orthonormal frame)

$$
\begin{aligned}
& \Gamma^{\hat{\mu}}=\gamma^{\hat{\mu}} \otimes \gamma_{(6)}, \quad \hat{\mu}=0, \ldots, 4, \\
& \Gamma^{\hat{\alpha}}=1 \otimes \gamma^{\hat{a}}, \quad \hat{a}=5, \ldots, 10,
\end{aligned}
$$

where the $\gamma^{\hat{\mu}}$ are $4 \times 4$ matrices generating an $S O(1,4)$ Clifford algebra, $\gamma^{\hat{a}}$ are $8 \times 8$ matrices generating an $S O(6)$ Clifford algebra and $\gamma_{(6)}=i \gamma^{\hat{5} \ldots \hat{10}}$.

In what follows, we will take a basis where $\gamma^{\hat{0}}, \gamma^{\hat{1}}, \gamma^{\hat{2}}, \gamma^{\hat{3}}$ are real (i.e. the usual fourdimensional Majorana representation). For $\gamma^{\hat{4}}$ we take $\gamma^{\hat{4}}=i \gamma^{\hat{0} \hat{1} \hat{2} \hat{3}}$, hence it is imaginary. The matrix $B$ will be of the form

$$
B=\gamma^{\hat{4}} \otimes B_{(6)} .
$$

The Calabi-Yau manifold on which we compactify has two covariantly constant $S O(6)$ spinors $\eta_{+}, \eta_{-}$. These can be taken to satisfy the following properties that will be useful later

$$
\begin{array}{ll}
\nabla_{a} \eta_{ \pm}=\nabla_{\bar{a}} \eta_{ \pm}=0 & \gamma_{(6)} \eta_{ \pm}= \pm \eta_{ \pm} \\
\gamma_{\bar{a}} \eta_{+}=\gamma_{a} \eta_{-}=0 & B_{(6)} \eta_{ \pm}=\mp i \eta_{\mp} \\
\left(\eta_{ \pm}\right)^{*}=\eta_{ \pm} & \rrbracket \eta_{ \pm}= \pm \frac{3 i}{l_{M}^{2} \tau_{2}^{1 / 3}} \eta_{ \pm} .
\end{array}
$$

We make the following ansatz for our eleven-dimensional Killing spinors

$$
\epsilon=\varepsilon^{1} \otimes \eta_{+}+\varepsilon^{2} \otimes \eta_{-},
$$

where $\varepsilon^{i}$ are spinors of the Clifford algebra in five dimensions. Using doublet notation for the five-dimensional spinors

$$
\varepsilon=\left(\begin{array}{l}
\varepsilon^{1} \\
\varepsilon^{2}
\end{array}\right)
$$

the eleven-dimensional Majorana condition (B.5) reduces to a symplectic Majorana condition for the five-dimensional spinors

$$
\varepsilon^{*}=\gamma^{\hat{4}} \otimes \sigma_{2} \varepsilon .
$$

We discuss the Killing spinor equations for the vacuum case (i.e. constant $\tau$ ), and afterwards we proceed to non-constant $\tau$ thus showing that the three-dimensional Gödel space we obtained is supersymmetric.

\section{The $\tau=$ constant case: the $\mathbf{A d S}_{3} \times \mathbf{S}^{2}$ vacuum}

\section{Killing spinors}

We first consider the case where $\tau$ is constant, when the geometry is locally $\mathrm{AdS}_{3} \times \mathrm{S}^{2}$. First we check the Killing spinor equations with the index in the Calabi-Yau space. From our decomposition (B.7) and the properties (B.6) we find that these equations are automatically 
satisfied. Next we look at the Killing spinor equations with an index in the five-dimensional space. With the choice of vielbein (hatted indices are frame indices)

$$
\begin{aligned}
e^{\hat{t}} & =\frac{l}{2 \tau_{2}^{1 / 3}}(d t+\chi), & & e^{\hat{\theta}}=\frac{l}{2 \tau_{2}^{1 / 3}} d \theta, \\
e^{\hat{z}} & =\frac{l}{2 \tau_{2}^{1 / 3}} e^{\phi} d z, & & e^{\hat{\varphi}}=\frac{l}{2 \tau_{2}^{1 / 3}} \sin \theta d \varphi, \\
e^{\hat{z}} & =\frac{l}{2 \tau_{2}^{1 / 3}} e^{\phi} d \bar{z}, & &
\end{aligned}
$$

(note the difference between the field $\phi$ and the angular coordinate $\varphi$ ) one finds, using (4.8), that the spin connection 1-forms are

$$
\begin{array}{ll}
\omega^{\hat{t} \hat{z}}=-\frac{i}{2} e^{\phi} d z, & \omega^{\hat{\theta} \hat{\varphi}}=-\cos \theta d \varphi, \\
\omega^{\hat{t} \hat{\bar{z}}}=\frac{i}{2} e^{\phi} d \bar{z}, & \\
\omega^{\hat{z} \hat{\bar{z}}}=-i(d t+\chi)+2(\partial \phi d z-\bar{\partial} \phi d \bar{z}) . &
\end{array}
$$

We find that the Killing spinor equations reduce to the following conditions on the fivedimensional spinor doublet $\varepsilon$ :

$$
\begin{array}{ll}
\left(\partial_{t}-i \gamma_{\hat{z} \hat{z}}\right) \varepsilon=0, & \partial_{\theta}\left(e^{-\frac{i \theta}{2} \gamma^{\hat{\varphi}}}\right) \varepsilon=0, \\
\left(\partial_{z}+\left(\partial \phi-i \chi_{z}\right) \gamma_{\hat{z} \hat{\bar{z}}}\right) \varepsilon=0, & \partial_{\varphi}\left(e^{-\frac{\varphi}{2} \gamma^{\hat{\theta} \hat{\varphi}}} e^{-\frac{i \theta}{2} \gamma^{\hat{\varphi}}}\right) \varepsilon=0 \\
\left(\partial_{\bar{z}}-\left(\bar{\partial} \phi+i \chi_{\bar{z}}\right) \gamma_{\hat{z} \hat{z}}\right) \varepsilon=0 . &
\end{array}
$$

Using the solution (4.17) for $\chi$, these are solved to give

$$
\varepsilon=\frac{\sqrt{l}}{\sqrt{2} \tau_{2}^{1 / 6}} e^{i(t+f) \gamma_{\hat{z} \hat{z}}} R(\theta, \varphi) \varepsilon_{0},
$$

where

$$
R(\theta, \varphi)=e^{\frac{i \theta}{2} \gamma^{\hat{\varphi}}} e^{\frac{\varphi}{2} \gamma^{\hat{\theta} \hat{\varphi}}},
$$

and $\epsilon_{0}$ is a constant five-dimensional spinor doublet. Note that the ambiguity $\chi \rightarrow \chi+d f$ induces a shift in the phase of the Killing spinors. The condition (B.9) imposes that $\varepsilon_{0}$ satisfies the symplectic Majorana condition

$$
\varepsilon_{0}^{*}=\gamma^{\hat{4}} \otimes \sigma_{2} \varepsilon_{0} .
$$

Hence we find 8 independent real Killing spinors. We see that our Killing spinors are antiperiodic when $z$ goes around a circle, indicating that we are in the Neveu-Schwarz sector, at least if we choose $f$ to have vanishing monodromy. This is the case in the coordinate systems we consider: both in the disc and upper half plane coordinate systems $(4.14,4.15)$, the function $f$ is zero.

\section{The $S U(1,1 \mid 2)_{L}$ algebra}

The solutions with $\tau$ constant have local Killing vectors obeying the algebra of $S L(2, \mathbb{R})_{L} \times$ $S L(2, \mathbb{R})_{R} \times S U(2)_{L}$. The $S L(2, \mathbb{R})_{L} \times S U(2)_{L}$ generators combine with the 8 Killing spinors derived above to form the the supergroup $S U(1,1 \mid 2)_{L}$. 
The $S L(2, \mathbb{R})_{L} \times S U(2)_{L}$ Killing vectors are

$$
\begin{array}{lll}
l_{0}=i \partial_{t}, & j_{3}=i \partial_{\varphi}, \\
l_{+}=2 e^{-\phi} e^{i(t+f)}\left(\chi_{z} \partial_{t}-\partial_{z}\right), & & j_{ \pm}=e^{\mp i \varphi}\left(i \partial_{\theta} \pm \cot \theta \partial_{\varphi}\right), \\
l_{-}=-2 e^{-\phi} e^{-i(t+f)}\left(\chi_{\bar{z}} \partial_{t}-\partial_{\bar{z}}\right), & &
\end{array}
$$

where $f$ is the arbitrary real function entering in the solution (4.17) for $\chi$.

We introduce a convenient basis for our Killing spinors (B.12) labelled by three doublet indices $m, \alpha, a$ taking values \pm 1 :

$$
g_{\frac{m}{2}}^{\alpha a}=\frac{\sqrt{l}}{\sqrt{2} \tau_{2}^{1 / 6}} e^{\frac{i m}{2}(t+f)} e^{-\frac{i \alpha \varphi}{2}} e^{\frac{i \theta}{2} \gamma^{\hat{\varphi}}} g_{0}^{\alpha a} \frac{m}{2},
$$

where the $g_{0 \frac{m}{2}}^{\alpha a}$ are constant spinors satisfying

$$
\begin{aligned}
\gamma_{\hat{z} \hat{z}} g_{0 \frac{m}{2}}^{\alpha a} & =\frac{m}{2} g_{0}^{\alpha a \frac{m}{2}}, \\
\gamma^{\hat{\theta} \hat{\varphi}} g_{0 \frac{m}{2}}^{\alpha a} & =-i \alpha g_{0}^{\alpha a \frac{m}{2}}, \\
\gamma_{(6)} g_{0}^{\alpha a} \frac{m}{2} & =a g_{0}^{\alpha a} \frac{m}{2} .
\end{aligned}
$$

In our explicit spinor basis, we take them to be

$$
\begin{aligned}
& g_{0}^{+a} \frac{m}{2}=\frac{1}{\sqrt{2}}\left(\begin{array}{llll}
0 & i & m & 0
\end{array}\right)^{T} \otimes \eta_{a}, \\
& g_{0} \frac{m}{2}=\frac{1}{\sqrt{2}}\left(\begin{array}{llll}
i & 0 & 0 & m
\end{array}\right)^{T} \otimes \eta_{a} .
\end{aligned}
$$

From the Killing vectors and Killing spinors one can compute the isometry supergroup of the supergravity background using the method developed in [22]. In particular, we are interested in the fermionic anticommutators, which can be computed by evaluating spinor bilinears. Defining fermionic anticommutators as

$$
\{g, \tilde{g}\} \equiv-i g^{T} C \Gamma^{M} \tilde{g} \partial_{M},
$$

where $C=B \Gamma^{0}$ is the charge conjugation matrix, we find the following superalgebra:

$$
\begin{aligned}
\left\{g_{\frac{m}{2}}^{\alpha a}, g_{\frac{m}{2}}^{\beta b}\right\} & =\epsilon^{\alpha \beta} \epsilon^{a b} l_{m}, \\
\left\{g_{\frac{m}{2}}^{\alpha a}, g_{-\frac{m}{2}}^{\beta b}\right\} & =\epsilon^{\alpha \beta} \epsilon^{a b} l_{0}+m \epsilon^{a b} T^{\alpha \beta},
\end{aligned}
$$

with

$$
T^{\alpha \beta}=\left(\begin{array}{cc}
-i j_{+} & j_{3} \\
j_{3} & -i j_{-}
\end{array}\right) .
$$

\section{M2-brane probes and their BPS properties}

Consider an M2-brane or anti-M2-brane probe, wrapped around the $\mathrm{S}^{2}$ and static with respect to the time $t$ in (4.3). Such a probe behaves as a point particle in $\mathrm{AdS}_{3}$ with action 
given in (3.9). From (B.15), we see the $l_{0}$ Noether charge (eigenvalue $L_{0}$ ) is the energy with respect to this time coordinate which is given by

$$
L_{0}=\frac{\pi l}{l_{3}} \equiv Z \text {. }
$$

We shall show that such a probe preserves half of the Killing spinors (B.12). The supersymmetry condition is

$$
\Gamma_{\kappa} \epsilon=\epsilon,
$$

where $\Gamma_{\kappa}$ is the idempotent operator that enters in the $\kappa$-symmetry transformations of the M2-brane [23, 24]. In our case it is given by

$$
\begin{aligned}
\Gamma_{\kappa} & = \pm i \Gamma_{\hat{t} \hat{\theta} \hat{\phi}} \\
& =\mp 2 i \gamma_{\hat{z} \hat{z}} \otimes \gamma_{(6)},
\end{aligned}
$$

where the upper (lower) sign corresponds to an (anti-)brane and we have used the properties of our basis of gamma matrices in the second line. Working out the SUSY condition a little further, we can write it as

$$
\pm 2 \gamma_{\hat{z} \hat{\bar{z}}} \sigma_{3} \varepsilon_{0}=\varepsilon_{0} .
$$

This is a good projection condition and is compatible with the Majorana condition (B.14); hence our probes leave 4 supersymmetries unbroken, with the antibrane preserving the opposite half of the brane. In terms of our basis (B.16), the preserved Killing spinors are

$$
\begin{array}{ll}
g_{\frac{1}{2}}^{\alpha+}, g_{-\frac{1}{2}}^{\alpha-} & \text { M2 - brane, } \\
g_{\frac{1}{2}}^{\alpha-}, g_{-\frac{1}{2}}^{\alpha+} & \text { anti - M2 - brane. }
\end{array}
$$

The 1/2-BPS projection correlates the $L_{0}$ eigenvalue with the internal chirality, but imposes no restriction on the R-symmetry quantum number $J_{3}$. The reason for the latter is that our $\mathrm{S}^{2}$-wrapped branes are invariant under R-symmetry.

We now investigate the supersymmetry properties of our probes from the point of view of the worldvolume superalgebra. Our branes wrap the nontrivial cycle $\mathrm{S}^{2}$ and carry a corresponding topological charge. As usual, this charge enters as a central extension in the superalgebra. We can compute the central terms using the results of [25]: the anticommutators (B.19) are modified to

$$
\{g, \tilde{g}\}=-i g^{T} C \Gamma^{M} \tilde{g} \partial_{M} \pm T_{M 2} \int_{S^{2}} \omega_{g, \tilde{g}}
$$

The closed two-forms $\omega_{g, \tilde{g}}$ can be computed by evaluating the spinor bilinears

$$
\omega_{g, \tilde{g}}=g^{T} C \Gamma_{\theta \varphi} \tilde{g} d \theta d \varphi .
$$

Doing this we find the extended superalgebra

$$
\begin{aligned}
\left\{g_{\frac{m}{2}}^{\alpha a}, g_{\frac{m}{2}}^{\beta b}\right\} & =\epsilon^{\alpha \beta} \epsilon^{a b} l_{m}, \\
\left\{g_{\frac{m}{2}}^{\alpha a}, g_{-\frac{m}{2}}^{\beta b}\right\} & =\epsilon^{\alpha \beta} \epsilon^{a b} l_{0}+m \epsilon^{a b} T^{\alpha \beta} \mp m Z \epsilon^{\alpha \beta} \sigma_{1}^{a b},
\end{aligned}
$$


where the (plus) minus sign corresponds to a (anti-) brane. This result agrees with [6], where it was derived from a construction of the worldvolume quantum mechanics. As discussed there, the short multiplets saturate a BPS bound

$$
L_{0} \geq j+Z
$$

and preserve $g_{\frac{1}{2}}^{\alpha+}, g_{-\frac{1}{2}}^{\alpha-}$ (for an M2-brane) or $g_{\frac{1}{2}}^{\alpha-}, g_{-\frac{1}{2}}^{\alpha+}$ (for an anti-M2-brane). This is consistent with the analysis above, and in particular we see that the Noether charge (B.22) is a consequence of the BPS bound for $j=0$.

\section{The $\tau$ nonconstant case: backreacted probes}

Consider the case where $\tau$ is not constant, where we showed that the local geometry becomes that of Gödel space. To check the supersymmetry of these solutions, we have to keep careful track of the additional terms in the susy variation (B.1) proportional to derivatives of $\tau$. For example, the spin connection receives extra contributions compared to (B.10)

$$
\omega=\omega_{(\text {B.10) }}+\alpha .
$$

One finds for the components of $\alpha$ :

$$
\begin{array}{rlrl}
\alpha^{\hat{t} \hat{z}} & =-\frac{i \bar{\partial} \bar{\tau} e^{-\phi}}{3 \tau_{2}}(d t+\chi), & & \alpha^{\hat{\varphi} \hat{z}}=-\frac{i \bar{\partial} \bar{\tau} e^{-\phi}}{3 \tau_{2}} \sin \theta d \varphi, \\
\alpha^{\hat{z} \hat{z}}=\frac{i}{3 \tau_{2}}(\partial \tau d z+\bar{\partial} \bar{\tau} d \bar{z}), & & \alpha^{\hat{a} \hat{z}}=\frac{i \bar{\partial} \bar{\tau} e^{-\phi}}{3 l \tau_{2}^{2 / 3}} e_{a}^{\hat{a}} d z^{a}, \\
\alpha^{\hat{\theta} \hat{z}}=-\frac{i \bar{\partial} \bar{\tau} e^{-\phi}}{3 \tau_{2}} d \theta, & & \alpha^{\hat{a} \hat{\bar{z}}}=-\frac{i \partial \tau e^{-\phi}}{3 l \tau_{2}^{2 / 3}} e_{a}^{\hat{a}} d z^{a},
\end{array}
$$

and mutatis mutandis for the complex conjugated components. Further $\tau$-derivatives enter in the expression for $\chi$ in (4.17) and in the 4-form field strength (3.5). Keeping track of all these terms and after tedious algebra, one finds that the backgrounds with nonconstant $\tau$ preserve Killing spinors precisely of the form (B.12) (with $\tau_{2}$ now of course a varying function), subjected to additional projection conditions:

$$
\begin{aligned}
& \gamma_{\hat{z}} \varepsilon_{0}^{1}=0, \\
& \gamma_{\hat{z}} \varepsilon_{0}^{2}=0 .
\end{aligned}
$$

These conditions project to one half of the supersymmetries, leaving 4 local Killing spinors. This is in precise agreement with our earlier probe analysis: we can combine the projections as

$$
2 \gamma_{\hat{z} \hat{\bar{z}}} \sigma_{3} \varepsilon_{0}=\varepsilon_{0} .
$$

In terms of our basis (4.13), the preserved Killing spinors are $g_{\frac{1}{2}}^{\alpha+}, g_{-\frac{1}{2}}^{\alpha-}$. Comparing with (B.26) we see that our backgrounds preserve the same supersymmetries as an $\mathrm{S}^{2}$-wrapped M2-brane probe and can hence be seen as backreacted geometries produced by such probe configurations. 


\section{Embedding in $5 \mathrm{D} \mathcal{N}=1$ supergravity}

We can interpret our system as a solution to $\mathcal{N}=1$ supergravity in five dimensions. We reduce the eleven-dimensional setup (3.2) to five dimensions on the Calabi-Yau threefold, giving rise to five-dimensional supergravity coupled to vector multiplets and one hypermultiplet (see for instance [64, 29]). We will also briefly sketch how the supersymmetry analysis is done in this setup, the details being analogous to the calculation of the previous section in eleven dimensions.

\section{Reduction to five dimensions}

Starting from the eleven-dimensional action (3.2), we can perform a reduction over the Calabi-Yau threefold only, using the ansatz (3.4). We are left with an action over five spacetime dimensions of the form

$$
\begin{aligned}
\frac{S_{5 d}}{2 \pi}= & \frac{1}{l_{M}^{3}} \int d x^{5} \sqrt{-g}\left(R-\frac{1}{2} \frac{\partial_{\mu} \tau \partial^{\mu} \bar{\tau}}{\tau_{2}^{2}}\right) \\
& -\frac{1}{2 l_{M}} \int G_{A B} F^{A} \wedge \star F^{B}+\frac{D_{A B C}}{6} \int A^{A} \wedge F^{B} \wedge F^{C} .
\end{aligned}
$$

In the reduction, we have kept the five dimensional metric, but we only allow the size $\tau_{2}$ of the internal manifold to vary, while the (internal) Calabi-Yau geometry is kept fixed. This leads to constant vector multiplet scalars $J^{A}$ in five dimensions, hence they do not appear in the action. The two-form field strengths $F^{A}$ are given in terms of the Kähler moduli $J^{A}$ as:

$$
F^{A}=\frac{l}{2 l_{M}} J^{A} \sin \theta d \theta \wedge d \phi,
$$

Note that the complex scalar $\tau$ is part of the universal hypermultiplet, for more information about the associated geometry, see [31].

We have shown how our setup is described in the framework of five-dimensional $\mathcal{N}=$ 1 supergravity, see for instance [64, 29] for a general discussion of its supersymmetric solutions. Moreover, as shown in the bulk of the paper, we have obtained a non-trivial solution of this system, with some of the hyperscalars turned on. Even though the general form of these solutions has been discussed before, not many explicit solutions with nontrivial hyperscalars are known.

\section{Supersymmetry analysis}

Consider the supersymmetry variations in five dimensions, as can be found in [29]. There are three equations which will concern us, from the variation of the gravitino, the gauginos and the hyperinos respectively. The gaugino variation is identically zero, due to the constant scalars and the special geometry properties. We are left with (all indices are flat):

$$
\begin{aligned}
0 & =\delta_{\epsilon} \psi_{M}^{i}=D_{M} \epsilon^{i}-\frac{i}{8} Y_{A} F^{A P Q}\left(\Gamma_{M P Q}-4 \eta_{M P} \Gamma_{Q}\right) \epsilon^{i} \\
0 & =\delta_{\epsilon} \zeta^{A}=i f_{X}^{i A} \Gamma^{M} \partial_{M} q^{X} \epsilon_{i},
\end{aligned}
$$


where $\psi_{M}^{i}$ are the gravitini, $\zeta^{A}$ the hyperini and $\Gamma$ are representations of the Clifford algebra in five dimensions. Note that the covariant derivative $D_{M}$ includes an important term from the quaternionic $S U(2)$ connection. Also, our gamma matrices are $i$ times the ones in [29] to take into account the signature change. We will use the equations above to look for BPS solutions.

Demanding the hyperino variation to vanish, leaves us with a projection condition making the solution 1/2-BPS. This projection condition is identical to (B.34). The BPS equation that follows from the gravitino equation is solved by our background. We will prove these two assertions below.

\section{Gravitino variation and BPS equations}

Using algebra similar to that in Sec. B, we see that the five-dimensional solution corresponding to the direct product of a sphere with constant radius and three-dimensional Gödel space, as given in $(3.4,4.21,4.22)$, indeed satisfies the gravitino equation. We conclude that we have a $1 / 2$-BPS solution to the equations of $\mathcal{N}=1$ supergravity in five dimensions.

\section{Hyperino variation and projection condition}

The supersymmetry variation of the hyperinos is given by (C.4). In general, the quaternionic manifold on which the hyperscalars live, has $S U(2) \times S p(n)$ holonomy, with $n$ the number of hypermultiplets. This holonomy structure is reflected in the index structure of the vielbein $f_{X}^{i A}$. In our case, only the universal hypermultiplet is excited. In the universal hypermultiplet $q^{X}, X=1 \ldots 4 n$, only the components corresponding to $\tau$ are nonzero. The quaternionic vielbein is given by [31]:

$$
f=\left(\begin{array}{cc}
0 & -v \\
\bar{v} & 0
\end{array}\right), \quad v=\frac{d \tau}{\tau-\bar{\tau}} .
$$

Then the hyperino equation gives rise to the following two conditions:

$$
0=\frac{\not \partial \tau}{\tau-\bar{\tau}} \epsilon^{1}, \quad 0=\frac{\not \partial \bar{\tau}}{\tau-\bar{\tau}} \epsilon^{2}
$$

If we now use that our solution for $\tau$ is holomorphic, we are left with two (different) projection conditions for $\epsilon^{1}, \epsilon^{2}$. These are the same projection conditions as in (B.34), implying that the solution we have is $1 / 2$-BPS.

\section{Israel junction conditions}

In this appendix we shortly review the derivation of the Israel junction conditions in a simple and transparent, but slightly unconventional way. We will give up some of the covariance of the original derivation [41], but in return the simplicity of our approach allows us to set up a formalism that is directly extended to more general field theories. Although in this appendix we choose an explicit set of convenient local coordinates it should be straightforward to rewrite our derivation in a fully covariant fashion. 
Notation: Before we get to the matching conditions themselves we start by introducing some notation and conventions.

Imagine a function $f(y)$ that is continous for all $y$ and smooth for all $y$ except at $y=y_{0}$. Then $f^{\prime}(y)$ is generically discontinuous at $y=y_{0}$ and $f^{\prime \prime}(y)$ has a delta-function singularity at $y=y_{0}$ proportional to

$$
\Delta f^{\prime} \equiv \lim _{y \rightarrow y_{0-}} f^{\prime}(y)-\lim _{y \rightarrow y_{0+}} f^{\prime}(y) .
$$

In this section and paper we will often find it useful to represent such functions as follows. A function $f$ with properties as above can formally always be written as

$$
f(y)=f_{+}(y) \Theta\left(y-y_{0}\right)+f_{-}(y) \Theta\left(y_{0}-y\right),
$$

where $f_{+}$and $f_{-}$are smooth functions and $\Theta$ is the Heaviside step function. Continuity at $y=y_{0}$ implies

$$
\Delta f \equiv \lim _{y \rightarrow y_{0}-} f(y)-\lim _{y \rightarrow y_{0+}} f(y)=f_{+}\left(y_{0}\right)-f_{-}\left(y_{0}\right)=0 .
$$

Furthermore, one then has

$$
\begin{aligned}
f^{\prime}(y) & =f_{+}^{\prime}(y) \Theta\left(y-y_{0}\right)+f_{-}^{\prime}(y) \Theta\left(y_{0}-y\right), \\
f^{\prime \prime}(y) & =f_{+}^{\prime \prime}(y) \Theta\left(y-y_{0}\right)+f_{-}^{\prime \prime}(y) \Theta\left(y_{0}-y\right)+\Delta f^{\prime} \delta\left(y-y_{0}\right),
\end{aligned}
$$

with generically $\Delta f^{\prime}=f_{+}^{\prime}\left(y_{0}\right)-f_{-}^{\prime}\left(y_{0}\right) \neq 0$.

Israel junction conditions: The Israel junction conditions put restrictions on the composition of a spacetime by 'gluing' two metrics along a hypersurface in a continuous way, i.e such that the induced metric on the hypersurface coincides for both metrics. If each of the metrics itself is a solution of the equations of motion then so is the composite spacetime, at least away from the hypersurface. On the hypersurface there will however generically be a non-vanishing singular term, as derivatives along a coordinate orthogonal to the hypersurface become singular there. As argued by Israel [41] one can cancel these singular terms in the e.o.m by introducing appropriate energy momentum also localized on the gluing hypersurface. This added energy-momentum then has a natural interpretation as the presence of a domainwall.

Let us derive the explicit form of the singular terms in the equations of motion. Locally we can always choose to write the metric under consideration in a set of Gaussian coordinates:

$$
d s^{2}=N^{2} d y^{2}+h_{a b} d x^{a} d x^{b} .
$$

These coordinates are chosen such that the hypersurface along which the two 'bulk' metrics will be glued, is simply given by $y=y_{0}$. By working in this specific set of coordinates we give up some of the full diffeomorphism invariance, but it improves the clarity of our discussion. We assume the components of the metric (D.6) to be made up of two different parts, coinciding at $y=y_{0}$ :

$$
N=N_{+} \Theta\left(y-y_{0}\right)+N_{-} \Theta\left(y_{0}-y\right), \quad h_{a b}=h_{a b}^{+} \Theta\left(y-y_{0}\right)+h_{a b}^{-} \Theta\left(y_{0}-y\right) .
$$


Although we assume the metric to be 'continuous', i.e. $\Delta h_{a b}=\Delta N=0$, it doesn't necessarily have to be smooth in $y$ at $y=y_{0}$.

Due to the second order nature of the Einstein equations it is clear that the Einstein tensor of such a 'glued' metric will be singular. It is a straightforward exercise to check that the Einstein tensor takes the form

$$
G_{\mu \nu}=G_{\mu \nu}^{+} \Theta\left(y-y_{0}\right)+G_{\mu \nu}^{-} \Theta\left(y_{0}-y\right)+G_{\mu \nu}^{(s)} \delta\left(y-y_{0}\right)
$$

with

$$
\begin{aligned}
G_{a b}^{(s)} & =-\frac{1}{N} \Delta\left(K_{a b}-h_{a b} K\right), \\
G_{a y}^{(s)} & =0 \\
G_{y y}^{(s)} & =0
\end{aligned}
$$

where $K_{a b}$ is the extrinsic curvature and $K$ its trace. It is clear that the two glued metrics each have to be solutions to the 'bulk' equations of motion for the composed metric to satisfy these equations; i.e. we also assume the energy momentum tensor takes the form

$$
T_{\mu \nu}=T_{\mu \nu}^{+} \Theta\left(y-y_{0}\right)+T_{\mu \nu}^{-} \Theta\left(y_{0}-y\right)+T_{\mu \nu}^{(s)} \delta\left(y-y_{0}\right) .
$$

Then the equations of motion at $y>y_{0}$ or $y<y_{0}$ take the form

$$
G_{\mu \nu}^{ \pm}=T_{\mu \nu}^{ \pm}
$$

However, as we derived above, the 'gluing' introduces a singular part to the Einstein tensor that still needs to be cancelled in the e.o.m. This is accomplished by having an appropriate $T_{\mu \nu}^{(s)}$, which can be interpreted as a domainwall with the correct effective tension. More precisely one can add to the Lagrangian a term of the form:

$$
L_{D . W .}=\delta\left(y-y_{0}\right) \tilde{L}_{D . W .} .
$$

It is then clear that the e.o.m coming from the standard Einstein-Hilbert Lagrangian with the domainwall added demand that

$$
\Delta\left(K_{a b}-h_{a b} K\right)=\frac{1}{\sqrt{-h}} \frac{\delta \tilde{L}_{D \cdot W .}}{\delta h^{a b}} \quad\left(\text { at } y=y_{0}\right) .
$$

This is our form of the Israel-junction conditions.

Generalised junction conditions: Using the notation and formalism introduced above it is straightforward to extend the Israel junction conditions to more general field theories. Take any field theory with a Lagrangian that only contains the fields and their first derivatives and a domainwall source that only couples to the fields and not their derivatives:

$$
L_{\text {total }}=L\left(\phi, \partial_{\mu} \phi\right)+\delta\left(y-y_{0}\right) \tilde{L}_{D . W .}(\phi) \text {. }
$$


If we again choose Gaussian coordinates on spacetime as in (D.6) and assume the fields to be glued ${ }^{8}$ at $y=y_{0}$, then it is straightforward to derive that the singular part is cancelled iff

$$
\Delta \frac{\delta L}{\delta \partial_{y} \phi}=\frac{\delta \tilde{L}_{D . W .}}{\delta \phi} \quad\left(\text { at } y=y_{0}\right) .
$$

Finally, note that the gravitational Israel-junction conditions (D.15) are a special case of this more general formula. To see this one has to use an equivalent gravitational Lagrangian that only involves first derivatives of the metric:

$$
L\left(g_{\mu \nu}, \partial_{\lambda} g_{\mu \nu}\right)=\sqrt{-g} g^{\mu \nu}\left(\Gamma_{\mu \alpha}^{\beta} \Gamma_{\nu \beta}^{\alpha}-\Gamma_{\mu \nu}^{\alpha} \Gamma_{\alpha \beta}^{\beta}\right) .
$$

One can then calculate that

$$
\frac{\delta L}{\delta \partial_{y} h_{a b}}=-\sqrt{-h}\left(K^{a b}-h^{a b} K\right)
$$

Applying formula (D.17) then gives exactly the Israel-junction conditions (D.15).

\section{References}

[1] E. Witten, Baryons and branes in anti de Sitter space, JHEP 07 (1998) 006, [hep-th/9805112].

[2] C. Bachas, M. R. Douglas, and C. Schweigert, Flux stabilization of D-branes, JHEP 05 (2000) 048, [hep-th/0003037].

[3] A. Simons, A. Strominger, D. M. Thompson, and X. Yin, Supersymmetric branes in AdS(2) $x$ $S^{* * 2}$ x CY(3), Phys. Rev. D71 (2005) 066008, [hep-th/0406121].

[4] J. Raeymaekers and K. P. Yogendran, Supersymmetric D-branes in the D1-D5 background, JHEP 12 (2006) 022, [hep-th/0607150].

[5] J. Raeymaekers, Open String Attractors, JHEP 04 (2007) 075, [hep-th/0702142].

[6] D. Gaiotto, A. Simons, A. Strominger, and X. Yin, D0-branes in black hole attractors, hep-th/0412179.

[7] D. Gaiotto, A. Strominger, and X. Yin, Superconformal black hole quantum mechanics, JHEP 11 (2005) 017, [hep-th/0412322].

[8] F. Denef, D. Gaiotto, A. Strominger, D. Van den Bleeken, and X. Yin, Black hole deconstruction, hep-th/0703252.

[9] E. G. Gimon and T. S. Levi, Black Ring Deconstruction, 0706.3394.

[10] S. R. Das et. al., Branes wrapping black holes, Nucl. Phys. B733 (2006) 297-333, [hep-th/0507080].

[11] E. Witten, Three-Dimensional Gravity Revisited, arXiv:0706.3359.

[12] W. Li, W. Song, and A. Strominger, Chiral Gravity in Three Dimensions, JHEP 04 (2008) 082, [arXiv:0801.4566].

\footnotetext{
${ }^{8}$ More precisely: $\phi=\phi_{+} \Theta\left(y-y_{0}\right)+\phi_{-} \Theta\left(y_{0}-y\right)$ with $\Delta \phi=0$.
} 
[13] B. R. Greene, A. D. Shapere, C. Vafa, and S.-T. Yau, Stringy Cosmic Strings and Noncompact Calabi-Yau Manifolds, Nucl. Phys. B337 (1990) 1.

[14] K. Godel, An Example of a new type of cosmological solutions of Einstein's field equations of graviation, Rev. Mod. Phys. 21 (1949) 447-450.

[15] D. Israel, Quantization of heterotic strings in a Goedel/anti de Sitter spacetime and chronology protection, JHEP 01 (2004) 042, [hep-th/0310158].

[16] J. P. Gauntlett, J. B. Gutowski, C. M. Hull, S. Pakis, and H. S. Reall, All supersymmetric solutions of minimal supergravity in five dimensions, Class. Quant. Grav. 20 (2003) 4587-4634, [hep-th/0209114].

[17] G. Compere, S. Detournay, and M. Romo, Supersymmetric Gódel and warped black holes in string theory, Phys. Rev. D78 (2008) 104030, [arXiv:0808.1912].

[18] E. G. Gimon and P. Horava, Over-rotating black holes, Goedel holography and the hypertube, hep-th/0405019.

[19] P. Berglund, E. G. Gimon, and T. S. Levi, Supergravity microstates for bps black holes and black rings, JHEP 06 (2006) 007, [hep-th/0505167].

[20] I. Bena and N. P. Warner, Bubbling supertubes and foaming black holes, Phys. Rev. D74 (2006) 066001, [hep-th/0505166].

[21] J. de Boer, F. Denef, S. El-Showk, I. Messamah, and D. Van den Bleeken, Black hole bound states in $A d S_{3} x S^{2}, 0802.2257$.

[22] J. P. Gauntlett, R. C. Myers, and P. K. Townsend, Supersymmetry of rotating branes, Phys. Rev. D59 (1999) 025001, [hep-th/9809065].

[23] E. Bergshoeff, E. Sezgin, and P. K. Townsend, Supermembranes and eleven-dimensional supergravity, Phys. Lett. B189 (1987) 75-78.

[24] E. Bergshoeff, R. Kallosh, T. Ortin, and G. Papadopoulos, kappa-symmetry, supersymmetry and intersecting branes, Nucl. Phys. B502 (1997) 149-169, [hep-th/9705040].

[25] E. J. Hackett-Jones, D. C. Page, and D. J. Smith, Topological charges for branes in M-theory, JHEP 10 (2003) 005, [hep-th/0306267].

[26] D. Gaiotto, A. Strominger, and X. Yin, The m5-brane elliptic genus: Modularity and bps states, hep-th/0607010.

[27] J. de Boer, M. C. N. Cheng, R. Dijkgraaf, J. Manschot, and E. Verlinde, A farey tail for attractor black holes, JHEP 11 (2006) 024, [hep-th/0608059].

[28] J. Raeymaekers, Near-horizon microstates of the D1-D5-P black hole, JHEP 02 (2008) 006, [arXiv:0710.4912].

[29] J. Bellorin, P. Meessen, and T. Ortin, All the supersymmetric solutions of $N=1, d=5$ ungauged supergravity, JHEP 01 (2007) 020, [hep-th/0610196].

[30] M. Huebscher, P. Meessen, and T. Ortin, Supersymmetric solutions of $N=2 d=4$ SUGRA: The whole ungauged shebang, Nucl. Phys. B759 (2006) 228-248, [hep-th/0606281].

[31] A. Strominger, Loop corrections to the universal hypermultiplet, Phys. Lett. B421 (1998) 139-148, [hep-th/9706195]. 
[32] K. Behrndt and S. Gukov, Domain walls and superpotentials from M theory on Calabi- Yau three-folds, Nucl. Phys. B580 (2000) 225-242, [hep-th/0001082].

[33] G. W. Gibbons, M. B. Green, and M. J. Perry, Instantons and Seven-Branes in Type IIB Superstring Theory, Phys. Lett. B370 (1996) 37-44, [hep-th/9511080].

[34] È. I. Semenov, New exact solutions to the nonautonomous liouville equation, Siberian Mathematical Journal 49 (2008), no. 1 166-174.

[35] I. Bengtsson and P. Sandin, Anti-de Sitter space, squashed and stretched, Class. Quant. Grav. 23 (2006) 971-986, [gr-qc/0509076].

[36] M. Rooman and P. Spindel, Goedel metric as a squashed anti-de Sitter geometry, Class. Quant. Grav. 15 (1998) 3241-3249, [gr-qc/9804027].

[37] D. Anninos, W. Li, M. Padi, W. Song, and A. Strominger, Warped AdS $S_{3}$ Black Holes, JHEP 03 (2009) 130, [arXiv:0807.3040].

[38] E. K. Boyda, S. Ganguli, P. Horava, and U. Varadarajan, Holographic protection of chronology in universes of the Goedel type, Phys. Rev. D67 (2003) 106003, [hep-th/0212087].

[39] N. Drukker, B. Fiol, and J. Simon, Goedel's universe in a supertube shroud, Phys. Rev. Lett. 91 (2003) 231601, [hep-th/0306057].

[40] V. Balasubramanian, E. G. Gimon, and T. S. Levi, Four Dimensional Black Hole Microstates: From D-branes to Spacetime Foam, JHEP 01 (2008) 056, [hep-th/0606118].

[41] W. Israel, Singular hypersurfaces and thin shells in general relativity, Nuovo Cim. B44S10 (1966) 1.

[42] J. R. David, G. Mandal, S. Vaidya, and S. R. Wadia, Point mass geometries, spectral flow and AdS(3)-CFT(2) correspondence, Nucl. Phys. B564 (2000) 128-141, [hep-th/9906112].

[43] M. A. Shifman and T. ter Veldhuis, Calculating the tension of domain wall junctions and vortices in generalized Wess-Zumino models, Phys. Rev. D62 (2000) 065004, [hep-th/9912162].

[44] J. Dai, R. G. Leigh, and J. Polchinski, New Connections Between String Theories, Mod. Phys. Lett. A4 (1989) 2073-2083.

[45] P. Horava and E. Witten, Heterotic and type I string dynamics from eleven dimensions, Nucl. Phys. B460 (1996) 506-524, [hep-th/9510209].

[46] D. Marolf and M. Trodden, Black holes and instabilities of negative tension branes, Phys. Rev. D64 (2001) 065019, [hep-th/0102135].

[47] S. D. Mathur, The fuzzball proposal for black holes: An elementary review, Fortsch. Phys. 53 (2005) 793-827, [hep-th/0502050].

[48] V. Balasubramanian, J. de Boer, S. El-Showk, and I. Messamah, Black Holes as Effective Geometries, Class. Quant. Grav. 25 (2008) 214004, [arXiv:0811.0263].

[49] S. D. Mathur, Fuzzballs and the information paradox: a summary and conjectures, arXiv:0810.4525.

[50] O. Lunin and S. D. Mathur, Ads/cft duality and the black hole information paradox, Nucl. Phys. B623 (2002) 342-394, [hep-th/0109154]. 
[51] S. Giusto and S. D. Mathur, Geometry of D1-D5-P bound states, Nucl. Phys. B729 (2005) 203-220, [hep-th/0409067].

[52] S. Giusto, S. D. Mathur, and A. Saxena, 3-charge geometries and their CFT duals, Nucl. Phys. B710 (2005) 425-463, [hep-th/0406103].

[53] S. Giusto, S. D. Mathur, and A. Saxena, Dual geometries for a set of 3-charge microstates, Nucl. Phys. B701 (2004) 357-379, [hep-th/0405017].

[54] I. Bena and N. P. Warner, One ring to rule them all ... and in the darkness bind them?, Adv. Theor. Math. Phys. 9 (2005) 667-701, [hep-th/0408106].

[55] V. S. Rychkov, D1-d5 black hole microstate counting from supergravity, JHEP 01 (2006) 063, [hep-th/0512053].

[56] J. de Boer, S. El-Showk, I. Messamah, and D. V. d. Bleeken, Quantizing N=2 Multicenter Solutions, arXiv:0807.4556.

[57] J. de Boer, S. El-Showk, I. Messamah, and D. V. d. Bleeken, A bound on the entropy of supergravity?, arXiv:0906.0011.

[58] M. Guica, T. Hartman, W. Song, and A. Strominger, The Kerr/CFT Correspondence, arXiv:0809.4266.

[59] G. Compere and S. Detournay, Centrally extended symmetry algebra of asymptotically Goedel spacetimes, JHEP 03 (2007) 098, [hep-th/0701039].

[60] A. Strominger and C. Vafa, Microscopic origin of the bekenstein-hawking entropy, Phys. Lett. B379 (1996) 99-104, [hep-th/9601029].

[61] J. Raeymaekers, W. Van Herck, B. Vercnocke, and T. Wyder, 5D fuzzball geometries and $4 D$ polar states, arXiv:0805.3506.

[62] D. Gaiotto, A. Strominger, and X. Yin, New connections between $4 d$ and $5 d$ black holes, JHEP 02 (2006) 024, [hep-th/0503217].

[63] J. P. Gauntlett and S. Pakis, The geometry of D = 11 Killing spinors, JHEP 04 (2003) 039, [hep-th/0212008].

[64] E. Bergshoeff et. al., $N=2$ supergravity in five dimensions revisited, Class. Quant. Grav. 21 (2004) 3015-3042, [hep-th/0403045]. 